www. revistad yo. com

\title{
Una revisión del estado del arte de los problemas asociados al transporte vertical me- diante ascensores en edificios
}

Pablo Cortés, Luis Onieva, Jesús Muñuzuri, José Guadix

Recibido: 1 de Febrero de 2018 / Aceptado: 14 de Febrero de 2018

\section{Resumen}

El transporte vertical es una disciplina que estudia los movimientos de personas en edificios. Los edificios altos se han convertido en una construcción común hoy en día. En dichos edificios, el transporte vertical es un problema que requiere un enfoque sistemático y ordenado. Así, para casos extremos en determinados edificios singulares, la ordenación del transporte vertical se convierte en un problema muy difícil de manejar, especialmente cuando diferentes personas llegan casi al mismo tiempo a plantas específicas deseando viajar hasta otras plantas de destino. Para resolver tales situaciones, la instalación de sistemas de control de grupos de ascensores (conocidos en inglés como Elevator Group Control Systems, EGCS) es una práctica habitual. Los EGCS se utilizan para gestionar ascensores coordinados múltiples en un edificio con el objeto de transportar pasajeros de manera eficiente. Los EGCS deben satisfacer las demandas asignando un ascensor a cada llamada de planta, realizando el despacho de ascensores atendiendo a diferentes criterios de optimización. Este artículo realiza una revisión sistemática y muestra distintas clasificaciones de las contribuciones más relevantes en la industria del transporte vertical, abordando tanto la revisión de la literatura científica, como las patentes en la industria y los trabajos recogidos en revistas de carácter profesional.

\section{Palabras clave}

Transporte vertical, ascensores, sistemas de control de grupos de ascensores, EGCS, despacho de ascensores, asignación, patrón de tráfico

\section{Antecedentes históricos}

Las fuentes históricas hacen referencia a diversos sistemas de elevación desde épocas antiguas. Así, en los textos del arquitecto romano Vitruvio, se señala que Arquímedes habría construido el primer sistema de elevación, en torno al año 236 a.C. También se dispone de fuentes que mencionan la existencia de ascensores conformados por plataformas sostenidas con cuerdas de cáñamo y accionadas a mano o por animales, que estaban instaladas en un monasterio de Sinaí (Egipto). Posteriormente, existe diversa bibliografía que acredita el desarrollo de de diferentes sistemas de elevación, mayormente diseñados para cargas pesadas, en distintos momentos de la historia.
No obstante, existe cierto consenso para asumir que la propuesta de elevador "seguro" de Elisha Graves Otis [1] en el año de 1852 (y comercializado a partir de 1853), como el nacimiento del ascensor "moderno". El ascensor disponía de un freno de emergencia que evitaba la caída libre de la cabina en caso de rotura del cable que la sostenía. Elisha demostró la eficacia de su sistema de emergencia instalando su ascensor en el edificio Crystal Palace de New York (Figura 1).

\footnotetext{
$\square$ Pablo Cortés

pca@us.es

Luis Onieva

onieva@us.es

Jesús Muñuzuri

munuzuri@us.es

José Guadix

guadix@us.es

Departamento de Organización Industrial y Gestión de

Empresas II. Escuela Técnica Superior de Ingeniería.

Universidad de Sevilla

Camino de los Descubrimientos s/n 41092- Sevilla (España)
} 
Figura 1 Demostración de un ascensor Otis Seguro en 1854.

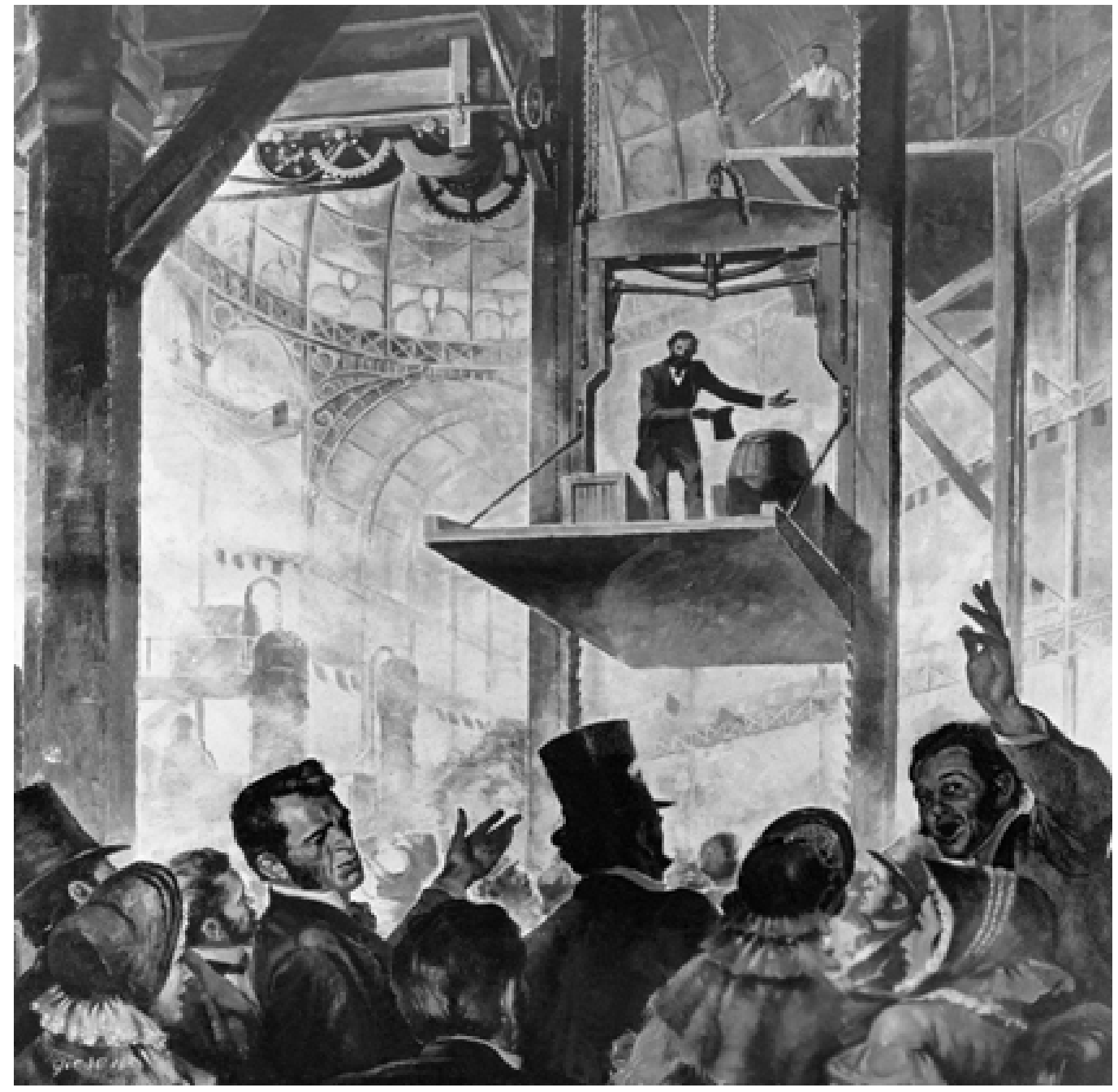

Tras la finalización de la II Guerra Mundial, en 1945, la construcción de edificios de altura media o alta se incrementó de forma muy significativa en todos los lugares del planeta. Incluso a finales del Siglo XX se desplegó una cierta carrera por la construcción del rascacielos más alto del mundo [3], la cual continúa en la actualidad (véase la Figura 2). 1880 durante la Exposición de Mannheim, aunque no fue hasta 1889 cuando Norton Otis desarrolló el primer ascensor eléctrico accionado mediante corriente continua. En 1900 se introdujo el motor de inducción para corriente alterna, siendo en 1903 cuando aparecieron en Estados Unidos los ascensores con corriente de tracción sin engranajes [2].

Figura 2 Edificios más altos del mundo a fecha de 2018.

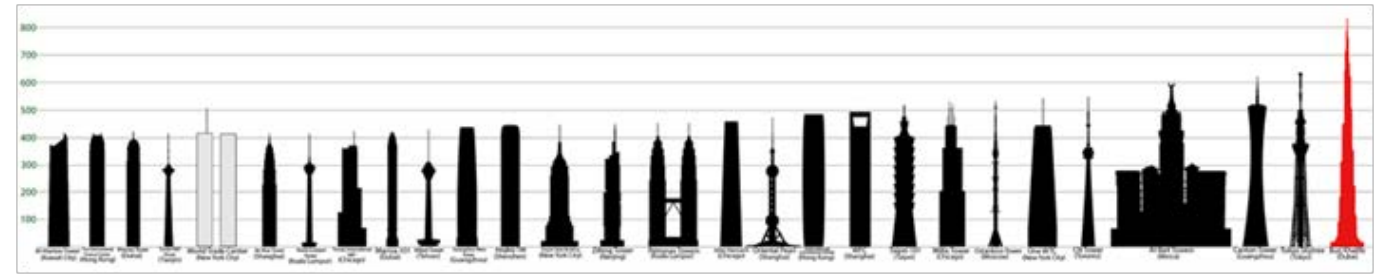


El desarrollo de ascensores eficientes, capaces de transportar a los pasajeros entre distintas plantas de un edificio, se convirtió de esta manera en una necesidad fundamental. Con ello, se incorporaron necesariamente tecnologías de automatización, control y electrónica, conforme estas fueron alcanzando la madurez, para la operación de los sistemas de trasporte vertical. En 1979 la Otis Elevator Company desarrolló el primer controlador basado en microprocesadores electrónicos, el cual mejoró ampliamente la eficiencia y flexibilidad de los sistemas de transporte vertical.

En la actualidad los sistemas de control de grupos de ascensores (conocidos en inglés como Elevator Group Control Systems, EGCS) constituyen el núcleo principal que gobierna la maniobra de los sistemas de transporte vertical. Dentro de estos sistemas, los algoritmos de despacho asignan en tiempo real, de modo altamente eficiente, las diferentes cabinas del grupo de ascensores a las distintas llamadas que puedan producirse en las plantas del edificio.

Si bien la literatura científica en el campo de investigación de los algoritmos de despacho en los sistemas de control de grupos de ascensores viene ganando una atención cada vez mayor, no se han publicado artículos de revisión con la misma proliferación que en otros campos de la disciplina de la Ingeniería de Organización ([4], [5]). La mayor fuente bibliográfica para la investigación científica en el campo se encuentra en las publicaciones de la sociedad IEEE (tanto en sus revistas como en sus congresos), si bien cada vez comienzan a aparecer más trabajos en publicaciones más afines a la Ingeniería de Organización (como son las revistas de los campos de Decision Science y Operations Research de los grupos editoriales Elsevier, Springer o INFORMS). En el campo profesional, las revistas Elevator World y Elevatori - The Elevator International Magazine son referencias inexcusables para cualquier investigador que desee iniciarse en el campo. Con relación a las patentes industriales, la empresa KONE Corporation ha desarrollado un papel de significativo liderazgo en los últimos tiempos, aunque también hay que destacar la labor de empresas como OTIS, Thyssen Krupp y, en menor medida, Schindler.

Este artículo consta de diez secciones. En la primera se ha realizado una introducción a los sistemas de transporte vertical. En la segunda se describen los sistemas de control de grupos de ascensores que gobiernan el transporte vertical mediante elevadores. En la tercera se muestra una taxonomía de los sistemas de control de grupos de ascensores. La cuarta sección revisa la literatura asociada al problema de captura de datos, mientras la quinta aborda el de detección de patrones de tráfico y la sexta la calibración de parámetros del sistema. La séptima sección recoge un detallado análisis del problema de asignación de cabinas a llamadas de planta (también conocido como problema de despacho de ascensores). La octava sección aborda la sectorización dinámica de edificios para mejorar los tiempos de servicio mediante ascensores dedicados a plantas. La novena sección incluye un análisis de arquitecturas de ascensores específicas diferentes a las tradicionales. Finalmente, la sección décima recoge una clasificación de las referencias y la undécima las conclusiones del trabajo y las líneas futuras de investigación.

\section{Los sistemas de control de grupos de ascensores}

Los EGCS disponen de los siguientes elementos debidamente integrados: una botonera de planta para poder solicitar ascensores (conocidas como llamadas de planta, y como landing call o hall call en la literatura inglesa), una botonera de cabina, desplegada dentro de cada uno de los distintos ascensores que tenga el grupo, para poder efectuar llamadas de cabina y señalar el destino al que se pretende viajar (conocidas como car call en inglés) y un controlador de grupo que incluirá los algoritmos de despacho y el controlador de la maniobra de cada ascensor.

La tarea primaria de un EGCS es la asignación eficiente de cabinas a llamadas de planta. El problema de realizar esta asignación surge cuando un pasajero realiza una llamada de planta en alguna de las del edificio. La asignación de una cabina u otra obedecerá a la satisfacción del criterio del algoritmo de despacho que incorpore el EGCS. Este podrá buscar minimizar los tiempos de espera de los pasajeros en cola para subirse al ascensor, minimizar los tiempos totales de viaje experimentados por los pasajeros (incluyendo los de espera en cola y los de viaje), minimizar el número de largas esperas en cola, maximizar la capacidad ocupada de los ascensores en movimiento, las rotaciones o rondas dadas al edificio por las diferentes cabinas, minimizar el consumo de energía del sistema, etc., o bien varios de ellos bajo un entorno de optimización multicriterio.

Por otra parte, los EGCS también deben tener en cuenta otros criterios, tales como la topología espacial del propio edificio, que depende fundamentalmente de los criterios constructivos del arquitecto y que, a diferencia de lo que suele ser la práctica habitual, también debería incorporar la opinión del diseñador del sistema de transporte vertical (actualmente existen distintas herramientas que permiten la planificación del sistema de transporte vertical en la fase constructiva, [6]). También, un elemento que condiciona el propio diseño del EGCS es la propia fisiología humana. Si bien no hay límite para la velocidad de viaje de los ascensores, sí se aconsejan limitaciones para los procesos de aceleración y deceleración, en las salidas y llegadas a planta, y al jerk experimentado (derivada de la aceleración). Actualmente, se consideran valores que no deberían superarse 1/8 $\mathrm{g} \times 1,5\left(\mathrm{~m} / \mathrm{s}^{\wedge} 2\right)$, para la aceleración y $2\left(\mathrm{~m} / \mathrm{s}^{\wedge} 3\right)$ para el jerk [7]. La Figura 3 muestra la curva de velocidad asociada al desplazamiento de ascensores. 
Figura 3 Variación de la velocidad y su variación respecto del tiempo en el desplazamiento de un ascensor.

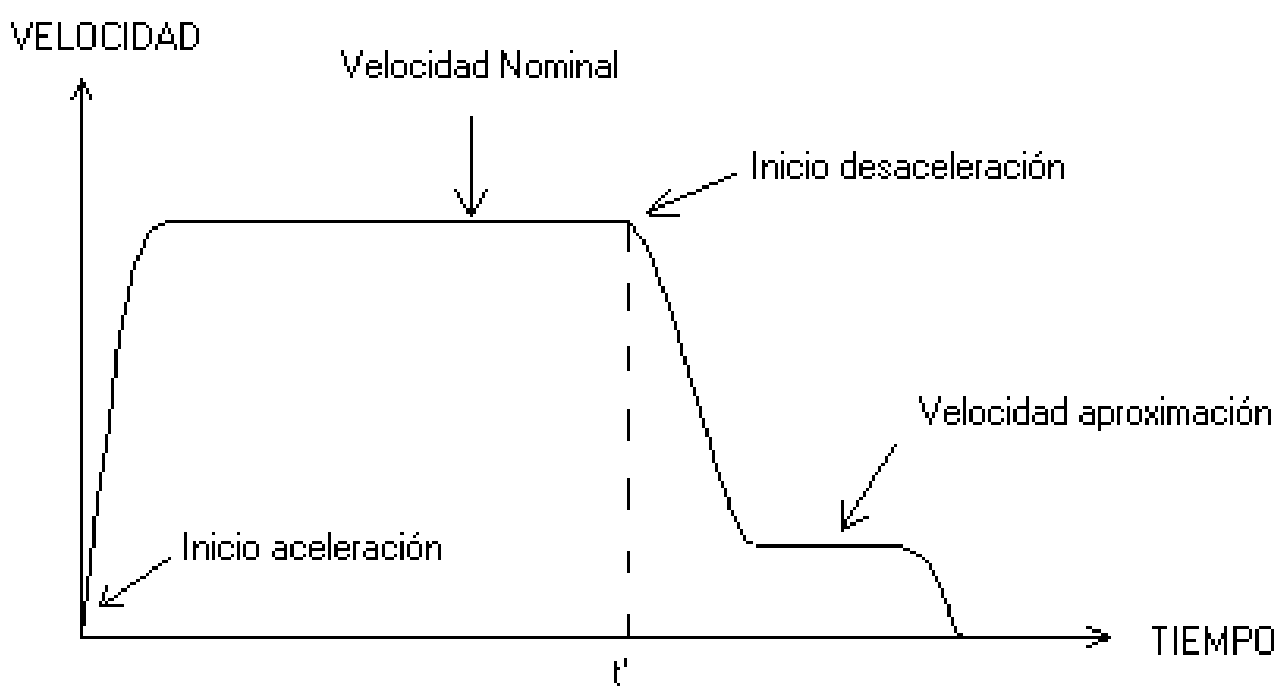

De igual forma, la psicología humana condiciona el diseño de los EGCS [8]. Aquí se incluyen los movimientos dentro del edificio, los procesos de llegada y salida del edificio, pero también las sensaciones experimentadas por los viajeros en las esperas, las cuales son más llevaderas dentro de la cabina que en cola en la planta, esperando la llegada del ascensor [9].

Un hecho adicional que afecta singularmente a los sistemas de transporte vertical y que condiciona los rendimientos de los EGCS es la existencia de la incertidumbre asociada al propio fenómeno. En efecto, cuando se produce la asignación de una cabina (que puede transportar además un grupo de viajeros en su interior, con viajes planificados para la cabina) ésta se realiza sin conocer el destino del pasajero que ha realizado la llamada de planta, pues solo lo indicará al entrar en el ascensor. La asignación se realiza (en el mejor de los casos) conociendo únicamente la dirección deseada de viaje. Aquí es necesario puntualizar que la mayoría de los ascensores son colectivos de bajada y subida, con botoneras dispuestas para grabar el movimiento deseado de viaje, pero que también existen sistemas con botoneras que no graban la dirección deseada de viaje (con lo que la incertidumbre es mayor), aunque también existen botoneras modernas que permiten incluso grabar el viaje de destino preseleccionándolo en origen en la botonera de planta (reduciendo en este caso la incertidumbre). La Figura 4 ilustra ejemplos de diferentes botoneras existentes en el mercado. Así, las imágenes (a), (b) y (c) representan botoneras con preselección del destino en el rellano; la imagen (d) muestra la preselección de la dirección del viaje (este es el tipo de botonera más común); la imagen (e) corresponde a una botonera de llamada de ascensor, en el que no se aporta información alguna relativa al movimiento que vaya a realizarse en el sistema, tan solo se señala la llamada de un ascensor; finalmente la imagen (f) ilustra una botonera de cabina. En caso de que la botonera de rellano fuese de preselección de destino (imágenes a, b ó c), no existiría botonera de cabina. La mayoría de los trabajos publicados han considerado sistemas con botoneras para sistemas colectivos de subida y bajada con preselección de dirección de viaje, que corresponden a la disposición más habitual en la industria. 
Figura 4 Imágenes de diferentes botoneras para llamadas de ascensores.

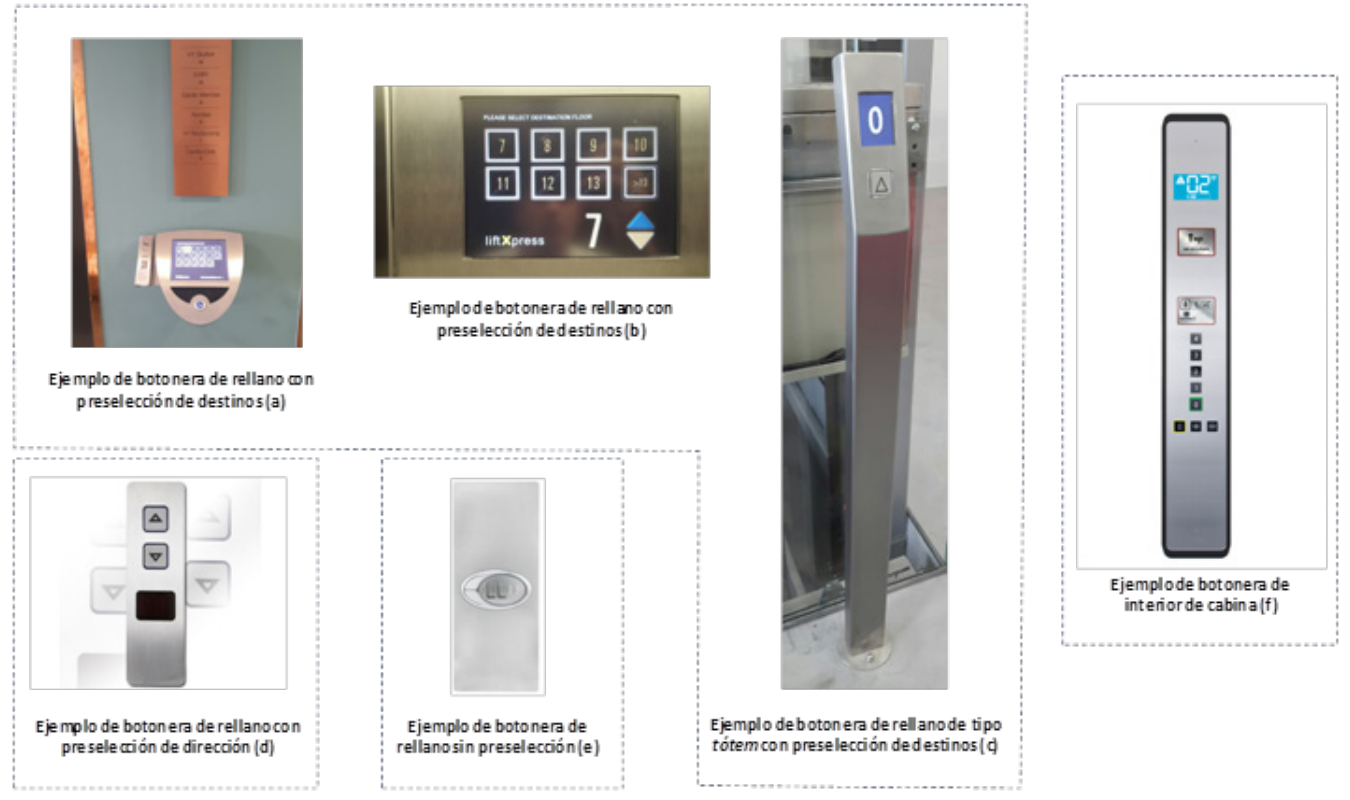

Finalmente, hay que resaltar el hecho de que la asignación de la cabina a la llamada de planta debe hacerse en tiempo real, disponiéndose de un tiempo muy limitado para proceder a tal asignación, lo que, dada la naturaleza NP-Dura del problema [10-11], hace complejo el diseño de los algoritmos de asignación y presenta aquellos enfoques basados en inteligencia artificial como especialmente atractivos.

\section{Taxonomía de los sistemas de control de grupos de ascensores}

En este apartado se incluye una taxonomía de los sistemas de control de grupos de ascensores, agrupando estos según sus tareas y sus funcionalidades.

La revisión de la literatura muestra cómo el problema que ha atraído más la atención científica es el de asignación de llamadas, probablemente por asociarse a los tradicionales y reglados problemas de secuenciación, profusamente estudiados desde la disciplina de la investigación operativa. Los criterios para proceder a la asignación han estado basados principalmente a los tiempos de espera de llegada de la cabina, es decir, a un modo de medición de la calidad de servicio experimentado por el viajero. Hay que destacar que, recientemente, el criterio de un uso eficiente de la energía del sistema de transporte vertical viene ganando interés en la comunidad científica. Por otra parte, también debe ser tenido en cuenta que, una cuestión que en numerosas ocasiones pasa a ser el criterio primario (en especial en grandes edificios con procesos de acceso de viajeros extraordinariamente elevados en fases concretas del día), es el de la optimización de la cantidad de servicio en lugar de la calidad de servicio. En estas situaciones, el objetivo es transportar cuantos más viajeros mejor en el menor tiempo posible. Para ello se recurre a estrategias de sectorización del edificio, asociando ascensores específicos a grupos de plantas concretas, especializando determinadas cabinas a los viajes entre ciertas partes del edificio. Además, estas zonas pueden variar a lo largo del día, dando lugar a sectorizaciones dinámicas. Existen, además, otros problemas específicos asociados a las tipologías de arquitecturas empleadas para los sistemas de transporte vertical, las cuales han venido creciendo de forma creativa en los últimos tiempos (esta casuística la estudiaremos en una sección específica posteriormente).

Por último, la incertidumbre asociada a todos estos procesos de asignación (desconocimiento de voluntades de viaje previamente al acceso a la cabina), ha llevado a otras investigaciones de interés ya que, en efecto, la calidad y la cantidad de servicio se verían significativamente mejoradas si se consiguiera reducir la incertidumbre asociada al proceso [12]. Esto se podría conseguir mediante procesos eficientes de captura de datos y de detección de patrones de tráfico en el sistema de transporte vertical, los cuales además permitirían el autoajuste de los procesos de asignación de llamadas a lo largo del día, adaptándolos al tráfico en el edificio.

Así las tareas de un sistema de control de grupos de ascensores se pueden dividir en dos grandes categorías que definiremos como primaria (para mejorar la calidad y/o la cantidad de servicio) y secundaria para mejorar los datos con los que trabajaran los algoritmos asociados a las tareas primarias (ver Figura 5). 
Figura 5 Taxonomía de las diferentes tareas de un sistema de control de grupos de ascensores

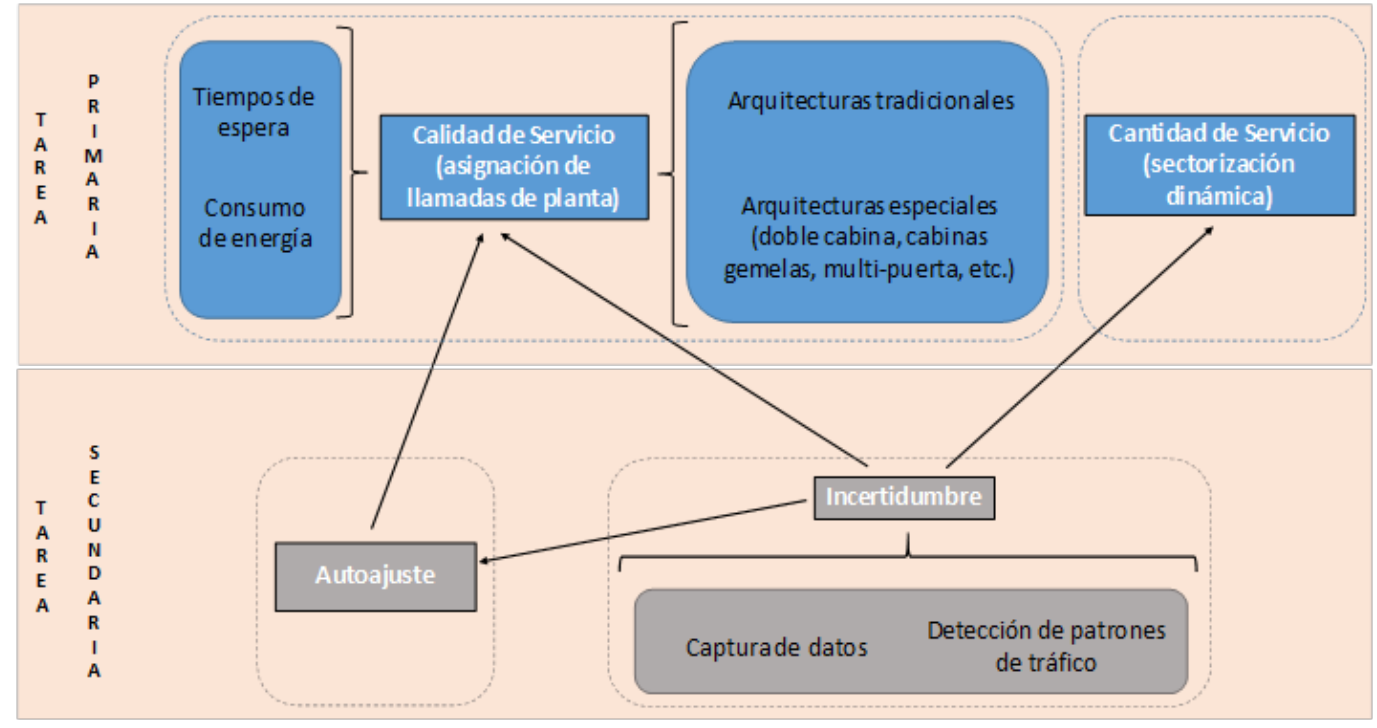

bloques modulares que puede incluir un EGCS para resolver los problemas reflejados en la Figura 5.
Mientras la Figura 5 representa las tareas de un EGCS y la interrelación entre dichas tareas, la Figura 6 muestra los
Figura 6 Interrelación entre los subsistemas de un EGCS

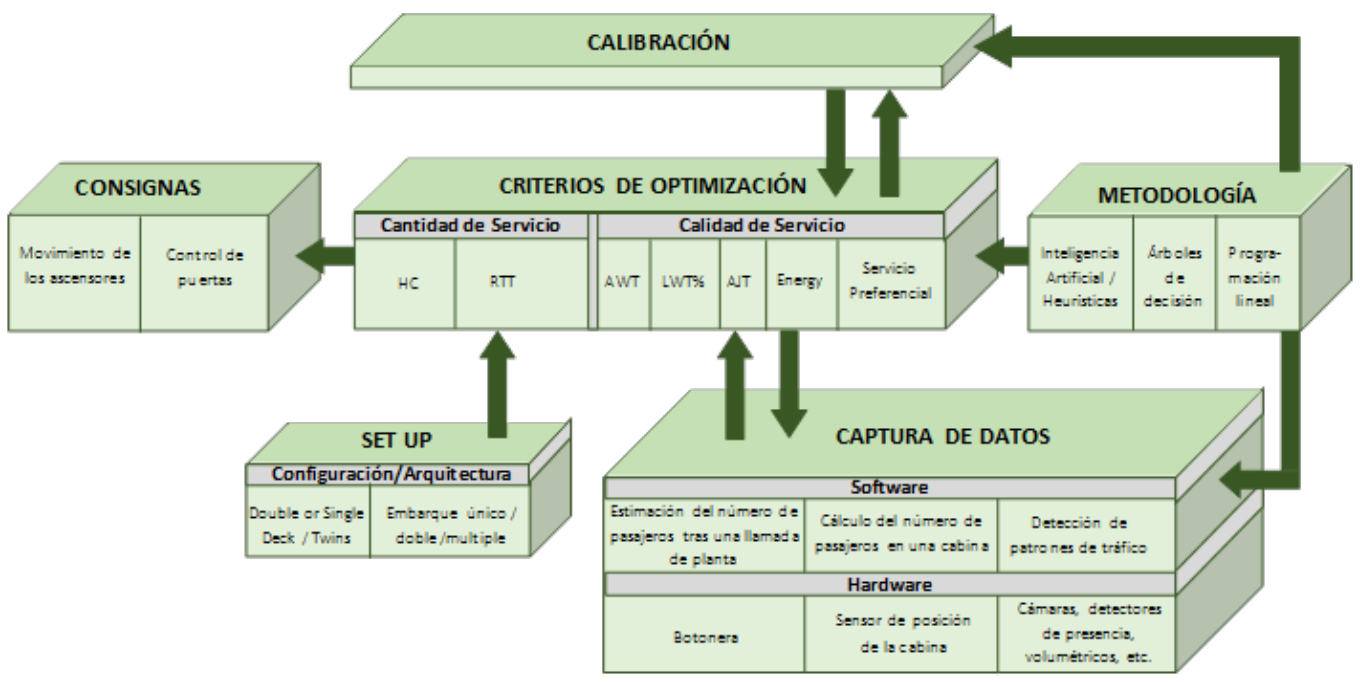

La capa de optimización pretende maximizar el servicio bajo diferentes criterios. Estos criterios pueden enfocarse a la mejora de la calidad de servicio del pasajero: minimizando los tiempos experimentados por los pasajeros con relación al tiempo medio de espera para la llegada del ascensor (AWT, Average Waiting Time), al tiempo medio de viaje dentro de la cabina (AJT, Average Journal Time) o al tiempo total en el sistema, que incluye la suma de los anteriores (ATT, Average Total Time). Aunque también puede emplearse como criterio la minimización del porcentaje de llamadas que experimentan largas esperas, típicamente más de 60 segundos y que se toma como una medida de la dispersión (\%LWT, Long Waiting Time Percentage). No obstante, hay que señalar que el AWT ha sido el criterio de calidad de servicio que más habitualmente se ha utilizado, debido a la percepción psicológica negativa de espera a la cabina en planta [9]. de tráfico reside en que existen criterios de optimización, y consecuentemente algoritmos, más eficientes dependiendo del patrón existente en el edificio. 
Hay que destacar que los gestores de edificios suelen incluir como medida relevante para la configuración de los EGCS el consumo de energía o, al menos, alguna forma de inclusión mediante una función objetivo multi-criterio. No obstante, y pese al interés industrial, pocos trabajos han incluido la consideración de la energía en la literatura científica [13]. En esta línea de investigación hay que destacar los trabajos que consideran la instalación de resistores capaces de recuperar energía cuando el contrapeso del sistema de elevación ayuda al propio movimiento del mismo en la direc- ción deseada [13-15] o la de aquellos orientados a conseguir reducciones de energía modificando las velocidades de los ascensores en función de carga y distancias [16]. Nótese que los ascensores están usualmente diseñados para que se mantengan en equilibrio con una carga igual a la mitad de la máxima admisible (que coincide con el peso del contrapeso del sistema de elevación). De esta forma, no todos los movimientos del ascensor consumen necesariamente energía, sino que incluso pueden generarla y devolverse para otros usos del edificio. La Figura 7 ilustra la descripción.

Figura 7 Situación de equilibrio en un ascensor considerando la carga transportada y el contrapeso

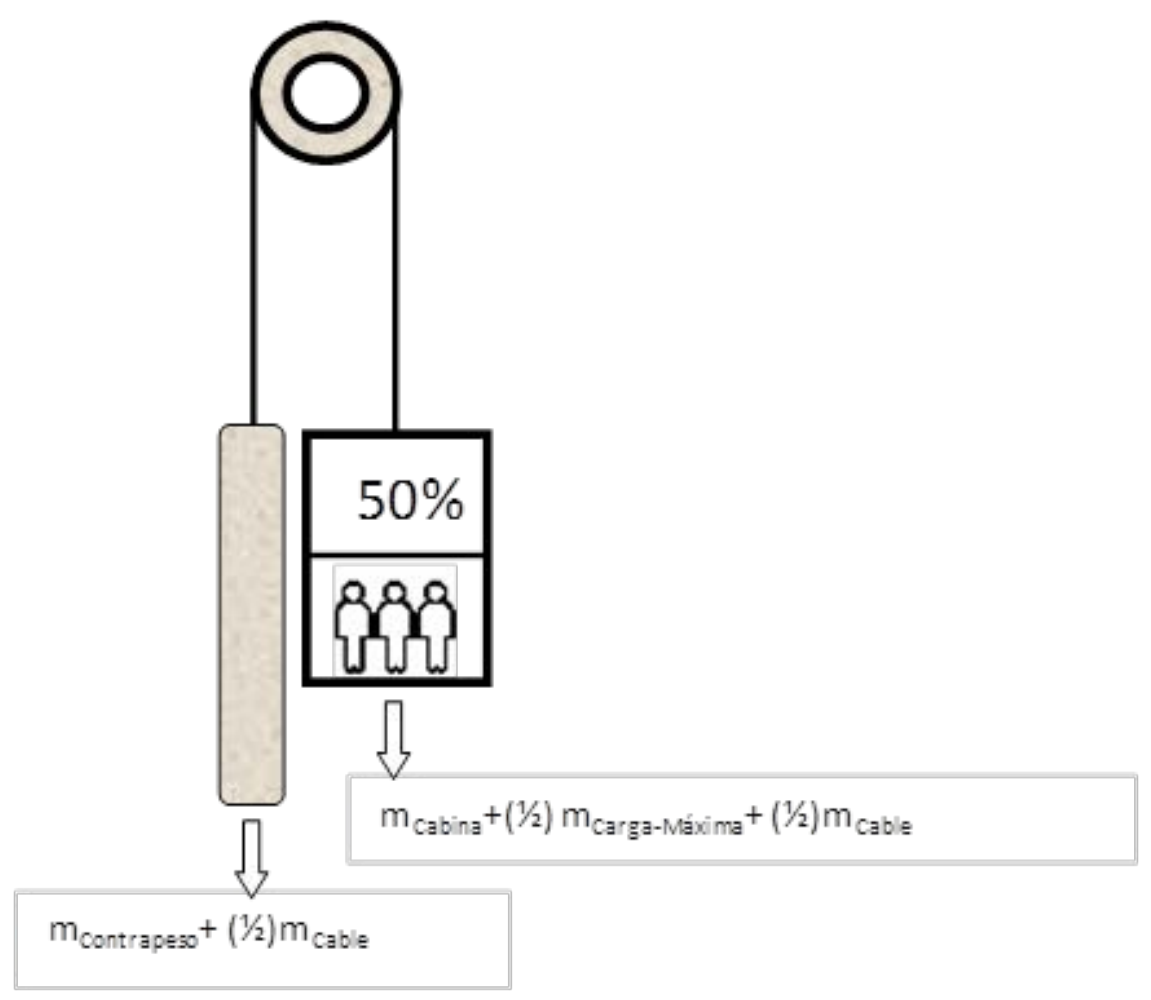

Por ser el problema de asignación de llamada de planta a cabina un problema de tipo NP-Duro, los principales enfoques de optimización se han basado en algoritmos de inteligencia artificial, aunque para casos de edificios pequeños se han ensayado soluciones mediante árboles de decisión o modelos de programación lineal. Industrialmente hay que reconocer que la mayoría de ascensores funcionan mediante simples (o no tan simples) heurísticas. Por otra parte, hay que recordar que los EGCS pueden autoajustarse (capa de calibración) recalibrando parámetros de los algoritmos o incluso cambiando de algoritmo, dependiendo de las condiciones del entorno (tipo de tráfico, información capturada por los dispositivos, etc.) y que dichos modelos y algoritmos deben configurarse para el tipo de instalación desplegada en el edificio (capa de set-up).

En las siguientes secciones se revisan los enfoques propuestos para abordar los distintos problemas que surgen asociados a los bloques constructivos o capas señalados en la Figura 4.

\section{Captura de datos}

La captura y posterior tratamiento de datos está directamente relacionado con las tareas de reconocimiento de patrones de tráfico y asignación de cabinas a llamadas de planta. El enfoque tradicional, hasta finales de la década de los 80 , fue recoger los datos por medio de observadores que hacían muestreos de tráfico en aquellos edificios que, por su tamaño, merecían un despliegue de este tipo [17]. A finales de esta década surgió la primera generación de EGCS que incorporaban microprocesadores permitiendo realizar ciertos cálculos estadísticos de tráfico.

Existen métodos diversos para calcular los pasajeros transportados entre plantas. Uno simple es mediante la resta de la carga inicial antes del viaje y la carga tras la parada (para lo cual la cabina debe contar tan solo con un pesacargas, actualmente obligado por normativa). A partir de aquí se extrapolan tablas semanales y diarias [18]. No obstante, el sistema es impreciso porque no identificaría un grupo de similar 
proporción entrando y saliendo en la planta. La instalación de detectores en los accesos a las cabinas en las plantas principales permite una toma de datos más eficiente y un mejor conocimiento de las colas de espera [19]. La instalación de cámaras, que puedan complementarse con algoritmos de reconocimiento de patrones, permitiría afinar aún más el número de pasajeros [20]. En este sentido, la utilización de cámaras con reconocimiento de imagen y por otro lado la instalación de sensores, son dos líneas abiertas contrapuestas, que se desarrollan de forma paralela en pugna por establecerse como la tecnología de referencia para identificar los pasajeros accediendo y viajando en las cabinas de los grupos de ascensores en la actualidad [21-23].

Finalmente, hay que destacar el significativo potencial que proporciona el conocimiento del destino de los pasajeros en el momento de la selección de la llamada. Esto se consigue con la instalación de botoneras de preselección de destino en los accesos a las cabinas de ascensores. [24].

\section{Reconocimiento de patrones de tráfi- Co}

Los patrones de tráfico se dividen en cuatro grandes grupos: tráfico predominante de subida (uppeak traffic en inglés), tráfico predominante de bajada (downpeak traffic), tráfico de mediodía (lunchpeak o midday traffic, donde se produce una mezcla de los tráficos uppeak y downpeak) y tráfico interplantas (interfloor traffic, que se produce para el tráfico del resto del día sin direcciones predominantes). La Figura 8 representa un típico patrón de tráfico para un edificio de oficinas en el cual se aprecian los movimientos de pasajeros a lo largo del día, observándose las llegadas coincidentes al inicio de la jornada laboral, salidas al final de la misma, una cierta fusión de tráfico uppeak y downpeak en el entorno del mediodía, dando lugar a un patrón midday o lunchpeak, y un tráfico interfloor para el resto de la jornada.

Figura 8 Patrón de tráfico en un edificio de oficinas

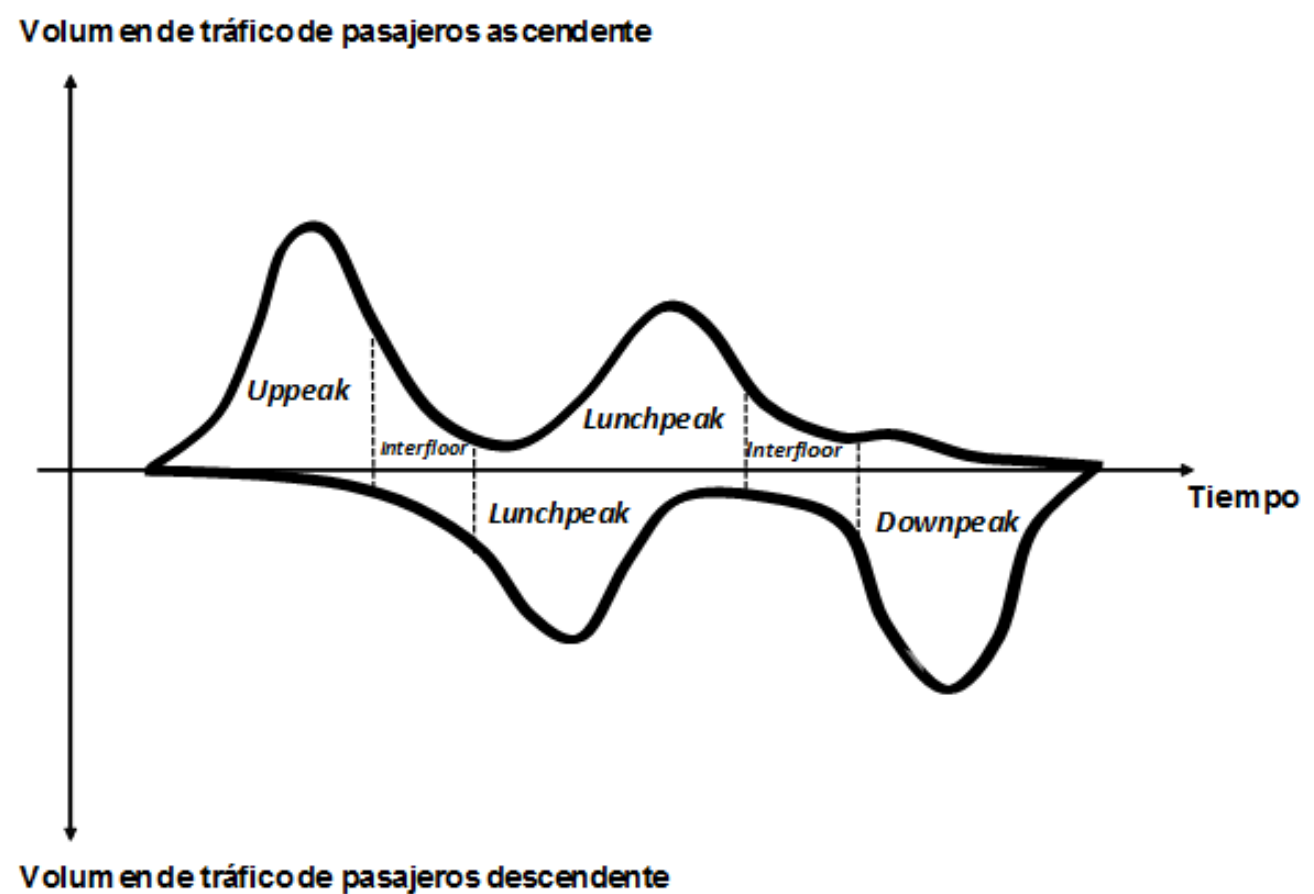

Varias razones justifican la realización de análisis rigurosos del tipo de tráfico del edificio. En primer lugar, destaca la posibilidad de poder seleccionar algoritmos de despacho ad hoc para el patrón de tráfico existente en un determinado periodo. En segundo lugar, porque muchos de los parámetros de desempeño empleados para del sistema, tales como el AWT o el tiempo de ronda (Round Trip Time, RTT), dependen de la correcta identificación del patrón (tal y como se señala en [7] y en los capítulos 3 y 4 de [17]). Así, por ejemplo, si no se identificara rápidamente el acceso a un patrón uppeak, se formarían largas colas en el hall de entrada del edificio incrementándose los tiempos de espera de los pasajeros significativamente. Es habitual en este tipo de patrones utilizar como criterio de desempeño del sistema el RTT, buscando generar numerosos viajes de ida y vuelta entre la planta baja y el reparto de pasajeros en las plantas del edifico, hasta el regreso del ascensor a la planta baja. Por otra parte, bajo este tipo de patrón los ascensores son forzados a volver a la planta baja vacíos. Si se funcionara bajo un patrón uppeak en una fase no uppeak, se estaría penalizando innecesariamente a pasajeros pretendiendo otro tipo de desplazamientos en el edificio. 
Sorprendentemente, el problema de reconocimiento de patrones es uno de los campos menos investigado en el transporte vertical. No obstante, también se ha realizado un progreso significativo desde las primeras propuestas exclusivamente basadas en la medición del porcentaje de carga movido en cada tramo horario, hasta complejas heurísticas capaces de identificar diferentes tipos de parones [25]. Los primeros pasos se dieron mediante metodologías que contrastaban mediciones realizadas con bases históricas de información. En [26], se propuso una base de datos histórica para reducir la incertidumbre mediante la grabación de eventos que se almacenaban con un periodicidad semanal, diaria y horaria. Este trabajo se mejoró con las aportaciones de [27]. En [28] se recogía una propuesta para determinar el comienzo y el final de los períodos de uppeak y downpeak. En esta propuesta, el número de accesos a las cabinas se comparaba con un umbral para detectar el patrón. Cuando el número de accesos superaba el umbral del tipo de tráfico correspondiente, se asociaba al patrón en cuestión. En general, se trataban de enfoques simples empleándose modelos basados en aprendizajes básicos [29], que se contrastaban con bases de datos históricas y recientes para determinar si el patrón de tráfico que se producía era de tipo uppeak, downpeak o ninguno.

Es habitual abordar los problemas de clasificación mediante el empleo de máquinas de vector de soporte. Por ello, las máquinas vectoriales de soporte mínimo cuadráticas también se han empleado para identificar los patrones de tráfico en transporte vertical [30,31].

Sin embargo, en los últimos tiempos la lógica difusa se ha mostrado como la herramienta más apropiada para la detección de patrones de tráfico. Uno de los primeros enfoques consistió en proponer reglas de lógica difusa para clasificar los patrones de tráfico, pero sin considerar ciertos datos de entrada relevantes, como la hora del día o las tasas de llegada y salida de pasajeros [32]. En [12], la detección del patrón de tráfico se plantea mediante un modelo de lógica difusa que soluciona la falta de memoria propia de los sistemas de control borroso considerando en las entradas el valor de la carga total ascendente y descendente durante un intervalo de tiempo, la variación de dicha carga ascendente y descendente entre períodos, y el patrón de tráfico detectado en el intervalo anterior, proporcionando resultados muy precisos para los patrones de ensayo tradicionales (conocidos como patrones CIBSE, Siikonen y Strakosch, véase [17]).

En ocasiones se ha recurrido a intentos más ambiciosos en los controladores difusos, tratando de que estos permitieran encontrar el tipo de tráfico y hacer a la vez propuestas de asignación de cabina a llamada de planta. Así, es la propuesta en [33], pero que carece de cualquier estrategia de control basada en criterios típicos, como el AWT, el porcentaje de largas llamadas en espera o el consumo de energía, haciéndolo de este modo poco fiable. Más interesante resulta la pro- puesta de [34] donde se propone un sistema de control que se puede dividir en tres módulos diferentes, uno para asignar llamadas, otro para generar la estrategia de control y otro para la gestión del tráfico, que reúne y memoriza el flujo de pasajeros con el tiempo.

\section{Calibración de parámetros}

Una de las principales dificultades a las que se enfrenta el diseñador del EGCS es el establecimiento a priori de valores numéricos para un elevado número de parámetros del sistema, que sin duda afectarán a su rendimiento [35]. Estos parámetros abarcan desde la propia configuración de algoritmos de optimización bio-inspirados, a los pesos asociados a heurísticas de despacho o simplemente a los parámetros que definen las regiones lingüísticas en los enfoques difusos.

Tradicionalmente, para establecer estos parámetros se ha venido recurriendo a expertos. No obstante, existe literatura científica que recoge esfuerzos importantes por realizar ajustes finos de este tipo de parámetros mediante métodos de calibración sistemáticos. Es el caso de la propuesta de [36] para calibrar los parámetros durante la noche, a partir de los datos recopilados durante el día. Este sistema lleva a cabo la calibración mediante el uso de un algoritmo genético cuyo objetivo es proporcionar robustez al sistema para que su rendimiento no se vea alterado al variar las condiciones de trabajo, como por ejemplo mediante cambios en la función objetivo (preferencias de los propietarios) o de otras condiciones de contorno. Estos cambios se abordan utilizando una memoria de soluciones pre-computadas. Sin embargo, la última tendencia en los sistemas de calibración consiste en resolver el problema sin inferir ningún dato de la memoria estadística [37-39].

En [37] se propone un algoritmo genético con una función muticriterio que incluye el AWT y el consumo de energía. En función de la demanda de viajes de pasajeros medida modifica los pesos de ponderación de la función objetivo multicriterio haciendo uso de un controlador PI.

En [38] se propone un sistema de calibración automático que considera la información procedente de datos históricos y estadísticos. La asignación de cabinas a llamadas de planta se realiza mediante un algoritmo borroso y cada cinco minutos el módulo de calibración compara el AWT medido con el que fue previsto, modificando los parámetros asociados a las funciones de membresía de los algoritmos difusos.

Por último, [39] recoge otra propuesta de auto-calibración, donde la asignación de cabinas a llamadas de planta se lleva a cabo mediante una red neuronal difusa calibrada mediante una combinación de un algoritmo de colonia de hormigas y un algoritmo de propagación hacia atrás. En primer lugar, el algoritmo de hormigas proporciona una solución global ráp- 
ida que considera los pesos de la estructura de red neuronal difusa como entradas y utiliza un algoritmo de propagación hacia atrás para realizar una búsqueda local exhaustiva y así obtener una solución global en el espacio de soluciones.

\section{El problema de despacho de ascen- sores o de asignación de cabinas a llamadas de planta}

Este problema surge cuando un pasajero, en un instante determinado, quiere viajar desde una planta de un edificio a otra planta diferente del mismo. El pasajero realiza una llamada a un ascensor presionando un botón de llamada de planta instalado en el rellano (o hall) y ubicada cerca de las cabinas del grupo de ascensores. Después de eso, el controlador del ascensor recibe la llamada e identifica cuál de las cabinas

Figura 9 Ilustración del proceso de asignación de cabina a llamada de planta del grupo de ascensores es la más adecuada para atender a la persona que ha emitido la llamada, mediante un algoritmo de despacho (también conocido como de asignación de cabina a llamada de planta). El EGCS tiene la tarea de controlar las llamadas desde dentro de cabina y de planta para controlar los movimientos de los ascensores con el objeto de que los pasajeros puedan ser transportados eficientemente a sus destinos. Así, el problema a resolver es seleccionar para cada llamada de planta una cabina, de forma que se minimice un criterio determinado, previamente establecido. La Figura 9 ilustra una situación en la que coexisten llamadas de planta para subir, bajar (identificadas con triángulos que señalan la dirección de viaje de la llamada de planta) y llamadas desde dentro de la cabina (identificadas mediante un círculo, marcando la planta a la que se dirigen los pasajeros, y que pueden ser más de una, como se aprecia en el ascensor que viaja en sentido descendente).

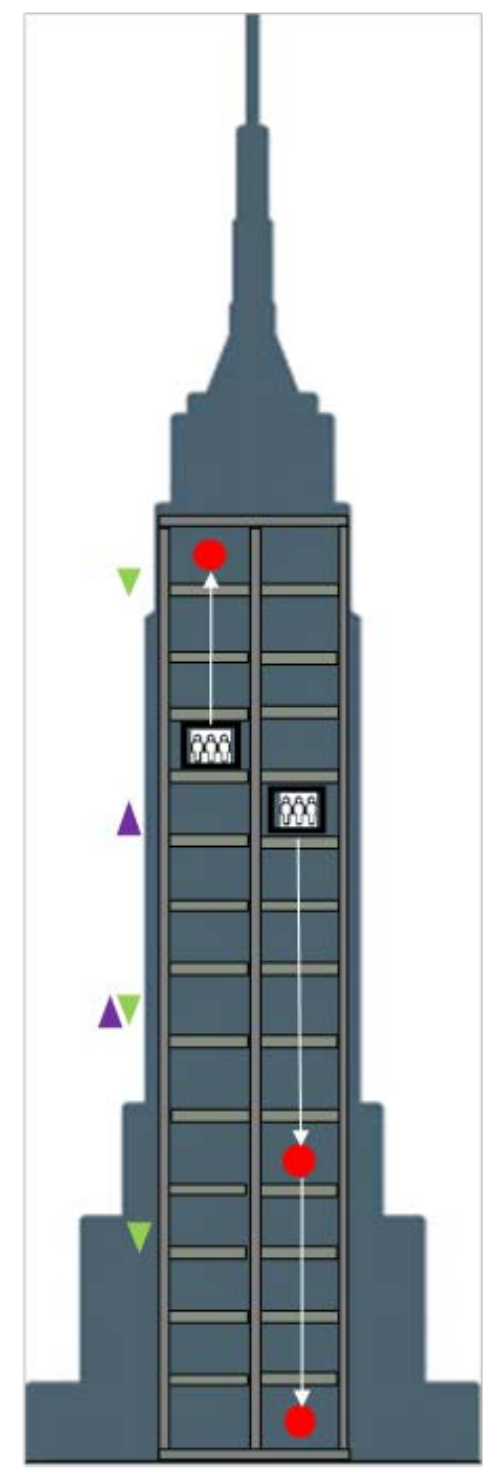

: ascensor con pasajeros : dirección de viaje del ascensar

: llamada de cabina

V : llamada descendente de planta

$\Delta$ : llamada ascendente de planta 
El algoritmo de asignación de cabinas a llamadas de planta o algoritmo de despacho constituye el núcleo fundamental del EGCS y es el que hace que éste sea más eficiente o menos. En la actualidad, las principales compañías de ascensores compiten duramente por alcanzar los diseños más sofisticados de algoritmos de despacho, los cuales protegen mediante patentes comerciales.

Como ya ha sido mencionado con anterioridad, el problema de asignación de cabinas a llamadas de planta es NP-Duro y el tiempo de computación requerido para alcanzar una solución se incrementa de forma no polinomial con el tamaño del problema. Por este motivo, los enfoques para la resolución de este problema han estado y están basados en heurísticas de despacho o en algoritmos basados en inteligencia artificial. En [40] se recoge, en este sentido, una extensa comparación de diferentes algoritmos de inteligencia artificial para la resolución del problema, estableciéndose una valoración del rendimiento de estos.

\subsection{Enfoques basados en heurísticas}

Los enfoques de despacho de ascensores basados en heurísticas siguen una serie de reglas lógicas simples que implementan un procedimiento de tipo greedy que consiga proporcionar una solución razonablemente buena. Este tipo de reglas son las que han tenido un mayor éxito de implantación en la industria (varias de ellas protegidas mediante patentes). La regla heurística más comúnmente implementada sigue como norma "realizar la asignación del ascensor más cercano que viaje en la dirección deseada por la llamada de planta”. Esta regla, o pequeñas variaciones de ellas es conocida como heurística de sistema colectivo, heurística THV, entre otros nombres (véase [7], [41], [42]).

En [43] se recoge un enfoque que realiza la asignación evaluando un algoritmo greedy que realiza una estimación de una función multicriterio evaluando el tiempo de espera, el tiempo de ronda, un factor de ocupación de las cabinas y considera la existencia de zonas preferenciales en el edificio. En un sentido similar, [44] incluye diversas funciones de coste que se evalúan de forma iterativa modificando las asignaciones de las llamadas de planta, mientras que [45] realiza la estimación del tiempo de espera mediante diferentes fórmulas que se ponderan a través de una función de coste específica. El enfoque seguido en [46] es diferente; en esta heurística se define una ventana temporal y tan solo se abordan las asignaciones que esperan ser resueltas en este intervalo de tiempo, manteniendo al resto en espera. De nuevo el enfoque considera diversos parámetros de desempeño para construir una función de evaluación compleja.

Otro enfoque [11], que incluimos dentro de esta sección, es el empleo de árboles de decisión que realiza procesos de ensayo de soluciones mediante técnicas de ramificación y acotación mediante criterios de desempeño. Dado el carácter no polinomial del proceso de búsqueda en árbol para el problema de despacho (el número de nodos y arcos aumenta exponencialmente en función del número de llamadas de planta pendientes, por lo que puede ser imposible alcanzar una solución óptima dentro del intervalo de tiempo especificado) la heurística delimita tal espacio estableciendo cotas de tiempo de espera previamente tabuladas para el edificio en cuestión y realizadas de forma ad hoc.

\subsection{Enfoques basados en lógica difusa}

La lógica difusa ha encontrado un gran ámbito de aplicación en el campo del transporte vertical por diferentes motivos. En primer lugar, la incertidumbre asociada a las llamadas que harán los pasajeros al entrar en la cabina conduce a tomar decisiones de asignación sin conocimiento de los datos (o con conocimiento limitado, esto es, solo la dirección del viaje). En segundo lugar, ciertos datos relevantes se conocen de forma imperfecta. Así, por ejemplo, los patrones de tráfico están generalmente asociados a tramos horarios, sin una comprobación correcta de la situación de tráfico real del edificio o, también, el conocimiento derivado de la ocupación de cabinas se extrapola directamente del peso de las mismas, siendo volumen y peso magnitudes que no son directamente equivalentes. Por otra parte, las implementaciones basadas en lógica difusa tienen la ventaja adicional de ser fácilmente implementables y de ser rápidamente computables.

La mayoría de los algoritmos de despacho basados en lógica difusa han sido de carácter estático. Una de las primeras aplicaciones dinámicas se realizó en [47], mientras que [48] recoge una implementación más reciente, aunque más simple. La diferencia entre una asignación estática y dinámica es que, en las dinámicas, las asignaciones de cabina a llamada de planta pueden ser revertidas, mientras que en las estáticas esto no es posible. Hay que tener en cuenta que, en el caso de implementar algoritmos dinámicos, estos no podrían coexistir con sistemas de notificación a los pasajeros que indicaran la cabina de llegada (pues esto generaría conflictos al pasajero, adicionales al ya de por sí vinculado a la propia espera, si la cabina de servicio fuese cambiando de forma dinámica en el tiempo).

Enfoques de carácter estático se han ensayado en [49] mediante un enfoque tradicional. En [50] se implementa una función difusa que evalúa los tiempos de espera y los de ronda, mientras que, en [15], la función de decisión se asocia al consumo energético en lugar de a los tiempos de espera. Por otra parte, [51] recoge un enfoque híbrido que integra lógica difusa y redes neuronales. Un número significativo de aplicaciones industriales de sistemas de despacho han sido también protegidas mediante patentes industriales por las compañías del sector [52-57]. Las diferencias radican en la modificación de los criterios de decisión que van desde los 
más tradicionales, hasta otros que permiten la introducción de reglas específicas por los gestores del edifico ([54], [55]). En línea similar se encuentra el trabajo de [58] donde las reglas de decisión difusas incluyen la existencia de plantas prioritarias definidas por el diseñador del sistema de transporte vertical.

\subsection{Enfoques basados en redes neuronales}

Los enfoques basados en redes neuronales gozaron de significativo interés en la última década del siglo pasado [59-63], coincidiendo con cierto auge de este enfoque. La principal ventaja de las redes neuronales es su capacidad para adaptarse a los cambios en el flujo de tráfico (sobre todo a largo plazo), mientras que su principal desventaja era el complejo diseño al que obligaba, implicando un mayor coste de implementación. Además, su capacidad de adaptación a cambios repentinos y abruptos en el flujo de tráfico se mostró como limitada. Tras el interés inicial, y debido a los problemas reseñados, las décadas siguientes reflejaron una pérdida significativa de interés en la aplicación de esta metodología a problemas de transporte vertical. El reciente resurgir de los enfoques neuronales, está atrayendo de nuevo el foco de la atención a esta metodología, o bien a implementaciones híbridas de la misma. Es el caso de la combinación de la lógica difusa con las redes neuronales que parece permitir resolver los problemas derivados de los cambios bruscos de patrones [64-66]. También la hibridación con otros métodos basados en algoritmos de inteligencia artificial, combinando la programación de redes genéticas (un tipo de algoritmo evolutivo, similar a los algoritmos genéticos, en el que los individuos se representan en los nodos de un grafo y los arcos suponen relaciones entre los individuos), los algoritmos de colonias de hormigas y las redes neuronales artificiales, ha sido propuesto para crear sofisticados enfoques [67].

\subsection{Enfoques basados en algoritmos genéticos}

Los algoritmos genéticos han sido ampliamente empleados en problemas de asignación. Sin embargo, su aplicación al transporte vertical, dado el carácter iterativo de estos, se consideraba ineficiente dado el alto consumo de tiempos de computación, muchas veces intrínseco a estos algoritmos ([68], [69]). Sin embargo, esto no fue motivo que impidiera la existencia contribuciones tanto en el campo académico como en el profesional [70-72].

Más recientemente y unido a la mejora de la capacidad de computación de los procesadores, la resolución del problema de asignación de cabinas a llamadas de planta mediante algoritmos genéticos se ha revelado como un enfoque capaz de ofrecer soluciones muy eficientes en un tiempo de computación limitado y admisible para la operación en tiempo real. En este sentido, [41] y [42] supusieron una de las prim- eras aportaciones que se realizaron para una definición eficiente del cromosoma de los individuos que facilitara tanto el procesado de los operadores genéticos, como un cálculo rápido de la aptitud (fitness) de los individuos. Ejemplos de este nuevo impulso a la aplicación de algoritmos genéticos son las propuestas [73] y [74], así como [37] donde investigadores de la KONE Corporation proponen algoritmos genéticos para resolver un problema multicriterio que tiene en cuenta tanto los tiempos de espera como el consumo de energía en el edificio.

\subsection{Otros enfoques computacionales de opti- mización}

En este apartado agrupamos otras propuestas para abordar el problema de despacho de ascensores mediante técnicas computacionales.

Los enfoques directos mediante programación dinámica [75] fueron considerados en un inicio. Pero la combinación de opciones en los procesos markovianos, en problemas que consideran un grupo coordinado amplio de ascensores viajando por edificios altos (sin llegar a considerar siquiera el nivel constructivo del rascacielos) haría inviable su operación en tiempo real, debido al tiempo requerido para la aplicación del algoritmo.

En [76] se sugiere modelar el sistema de transporte vertical como una red de Petri. Las redes de Petri son una generalización de la teoría de autómatas que a través de una representación gráfica de un sistema de eventos discretos permite describir la topología de un sistema distribuido, paralelo o concurrente, tal como el que acontece en el transporte vertical. Con respecto al modelo de transporte vertical, la red puede estar representada por lugares, transiciones, arcos dirigidos y marcas o hitos que ocupan ciertas posiciones en la red. Esas marcas se identifican para el seguimiento de los viajes alrededor de la red, representando a los pasajeros y las cabinas. Cada posible variación de la red de Petri representa una posible asignación. Con el mismo enfoque, en [77] se propone que una red de Petri asociada a un sistema de transporte vertical conduce a un sistema delimitado que se puede resolver usando un algoritmo de árbol de Karp-Miller, si bien consumiría un tiempo de computación no polinomial.

Por otra parte, en el campo de la inteligencia computacional, además del protagonismo experimentado por los algoritmos genéticos ya señalados en el apartado anterior, se han desarrollado también otros enfoques tanto de corte bio-inspirado, como de búsqueda por trayectoria. En general, estos enfoques tienen las mismas dificultades que las especificadas para los algoritmos genéticos, relativas a la convergencia en tiempo real, así como también sus ventajas asociadas, como son una adecuada búsqueda en el espacio de soluciones capaz de proporcionar propuestas de despacho eficientes. Es el 
caso de aplicaciones basadas en algoritmos de optimización mediante enjambres de partículas (Particle Swarm Optimization, PSO) [78]; algoritmos basados en sistemas víricos (Viral Systems, VS) [79]; algoritmos de optimización basados en colonias de hormigas (Ant Colony Optimization, ACO) [80] o algoritmos basados en algoritmos de búsqueda tabú (Tabu Search, TS) [81].

\subsection{Enfoques basados en sistemas multiagente}

Los sistemas multiagente aparecen como oposición a los sistemas de control centralizados y constituyen una forma de control autónoma basada en agentes independientes que se pueden intercomunicar mutuamente y que toman decisiones a través de un proceso de aprendizaje. En general, estas decisiones pueden tomarse en régimen de cooperación o competición. En el caso del transporte vertical, los agentes se asocian a las diferentes cabinas de ascensores presentes en el grupo coordinado que actúa de forma cooperativa. La principal ventaja de un sistema descentralizado de control de ascensores es su robustez frente a fallos y su capacidad de adaptarse a las fluctuaciones del tráfico. La asignación de cabinas a las llamadas de planta se lleva a cabo mediante dos procesos diferentes: uno vinculado al proceso de aprendizaje y otro asociado a las reglas de despacho.
En el ámbito de esta tecnología las contribuciones son aún incipientes [82-84] pero su aplicabilidad emerge como un campo de trabajo prometedor.

\section{El problema de gestión de la sector- ización dinámica}

Uno de los enfoques más eficientes para aumentar el volumen de pasajeros movilizados por el sistema de transporte vertical (Handling Capacity, en la literatura especializada) durante los momentos de tráfico uppeak severos es la sectorización (o división) del edificio en zonas que atiendan cabinas específicamente asignadas. Esta medida permite reducir, además, los tiempos de ronda y también los tiempos de espera de los pasajeros. Evidentemente, la eficiencia de un sistema de sectorización dinámica estará asociado al rendimiento de los sistemas de detección de tráfico. La Figura 10 representa gráficamente la sectorización de un edificio.

Figura 10 Ejemplo de sectorización en un edificio

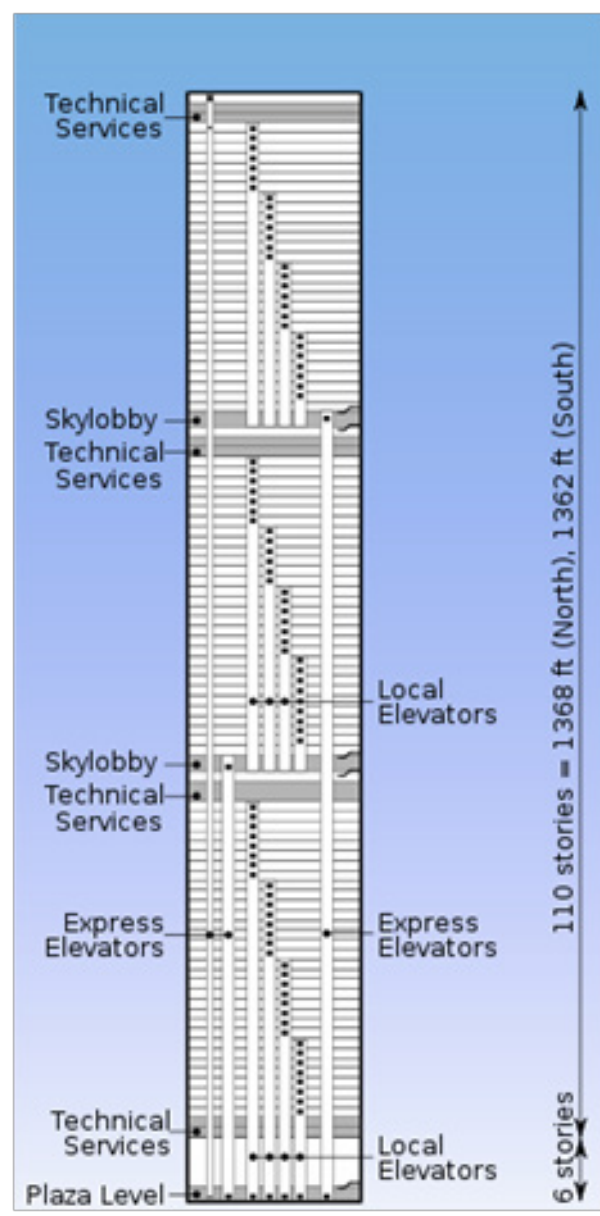


A grandes rasgos, los métodos de sectorización se pueden clasificar como estáticos o dinámicos, según sean los límites de las zonas fijos o flexibles, respectivamente. La sectorización dinámica funciona mejor porque es capaz de adaptarse a los cambios en el flujo de tráfico. A diferencia de la estática, la sectorización dinámica necesita implementar heurísticas ad hoc o algoritmos de inteligencia artificial para obtener un rendimiento eficiente. Por el contrario, en el caso de la sectorización estática [85], los métodos exactos pueden funcionar razonablemente bien pues el número de casos a evaluar viene acotado, no creciendo no-polinomialmente. Es por ello por lo que el interés para nosotros se centra en la sectorización dinámica.

Los primeros enfoques se encuentran en propuestas basadas en métodos de programación dinámica [86-87], que presentan las dificultades de exploración de este tipo de procesos markovianos en problemas de grandes dimensiones.

Más recientemente, la aplicación de sistemas inmunes ha dado resultados exitosos ([88], [89]). En los sistemas inmunes, el proceso de búsqueda para encontrar una solución se desarrolla de manera similar al comportamiento de los sistemas inmunes naturales, donde el antígeno es la función objetivo a optimizar (el tiempo de ida y vuelta de la cabina) y el problema de optimización es el anticuerpo. La afinidad entre el antígeno y el anticuerpo se decide de acuerdo con el valor de la función objetivo y la semejanza entre los anticuerpos se decide según el parecido entre las soluciones. La excitación entre los anticuerpos promueve una búsqueda más rápida en torno al óptimo, mientras que las células de memoria evitan el desperdicio innecesario del tiempo de ejecución.

Los algoritmos de optimización basados en enjambres de partículas también se han aplicado con éxito al problema [9091]. En estos algoritmos cada solución se codifica como una partícula (vector espacial) y tiene su propia velocidad asociada (también un vector) que define su movimiento a través del espacio de solución durante cada iteración. Después de cada iteración, la velocidad y la trayectoria de la partícula se ajusta de acuerdo con la distancia entre su mejor posición y la mejor posición alcanzada por todo el enjambre.

\section{Diseño de la arquitectura de sistema de transporte vertical}

En esta sección se revisan otras arquitecturas de transporte vertical alternativas a las tradicionales, como es el caso de los ascensores double-deck (que hoy en día tienen ya un grado de despliegue bastante significativo, en especial en los países asiáticos) y los ascensores gemelos (twin elevators).

\subsection{Sistemas double-deck}

Un sistema de elevación double-deck consiste en dos cabinas de ascensor unidas para poder ofrecer un servicio paralelo, al mismo tiempo, en dos plantas adyacentes. La Figura 11 muestra unos ascensores double-deck en el Midland Square, en Nagoya (Japón). Se ha señalado que los ascensores double-deck pueden conseguir disminuir la huella de carbono entre un $25 \%$ y un $40 \%$ [17].

En general, los ascensores double-deck, han demostrado mejores rendimientos para determinados patrones de tráfico (típicamente en uppeak, mejorando la handling capacity y el round trip time), pero pueden producir peores resultados para otros tráficos, o incluso conflicto entre los tiempos de espera (mejorándolos) y los tiempos de viaje (empeorándolos) [92].

Figura 11 de los ascensores double-deck

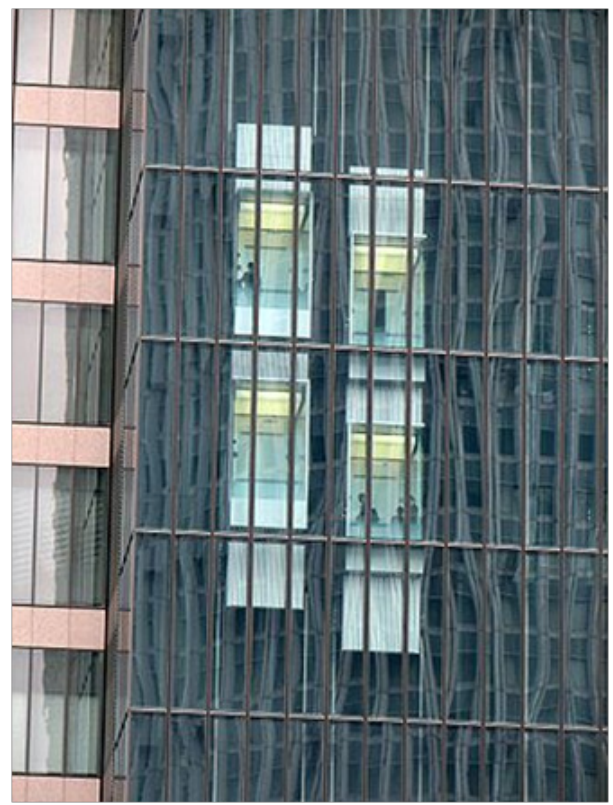


Los primeros double-deck datan de la década de los años 60 cuando OTIS desarrolló un sistema double-deck muy rudimentario, que consistía en una cabina principal y un tráiler que ésta remolcaba. Asimismo, utilizaba como algoritmo de despacho un sistema colectivo en subida y bajada bastante primario.

La evolución de algoritmos específicos para los sistemas double-deck ha seguido un proceso similar al experimentado por los ascensores de arquitectura habitual. Así, a finales del siglo pasado era común encontrar aplicaciones de tipo neuronal [93-94]. También heurísticas específicas han sido propuestas por algunas de las principales compañías y registradas mediante patentes [95-98]. Enfoques más sofisticados, mediante la implementación de algoritmos genéticos han sido también desarrollados [99-100]. Implementaciones genéticas se han ensayado también en [101] y [102], si bien el estudio de los resultados producidos por estos algoritmos incorpora de nuevo ciertas dudas sobre esta arquitectura

Figura 12 Ascensores gemelos de tipo twin para determinadas situaciones. Así, parece perjudicar los rendimientos del sistema de transporte vertical bajo tráfico downpeak y, por el contrario, los beneficios no serían tan significativos en el periodo uppeak. Más entusiastas son las conclusiones derivadas de los trabajos en [103] y [104] donde también se implementaron algoritmos de tipo evolutivo, siendo el [103] hibridado con un algoritmo de hormigas.

\subsection{Ascensores gemelos (twin elevators)}

A diferencia del caso de los double-deck, esta disposición considera dos cabinas desplazándose en un mismo hueco de ascensores. Lógicamente este tipo de disposiciones requiere mecanismos de control adicionales que eviten el conflicto en los desplazamientos y los riesgos asociados a hipotéticas colisiones entre las cabinas. La Figura 12 ilustra la configuración de ascensores twin.

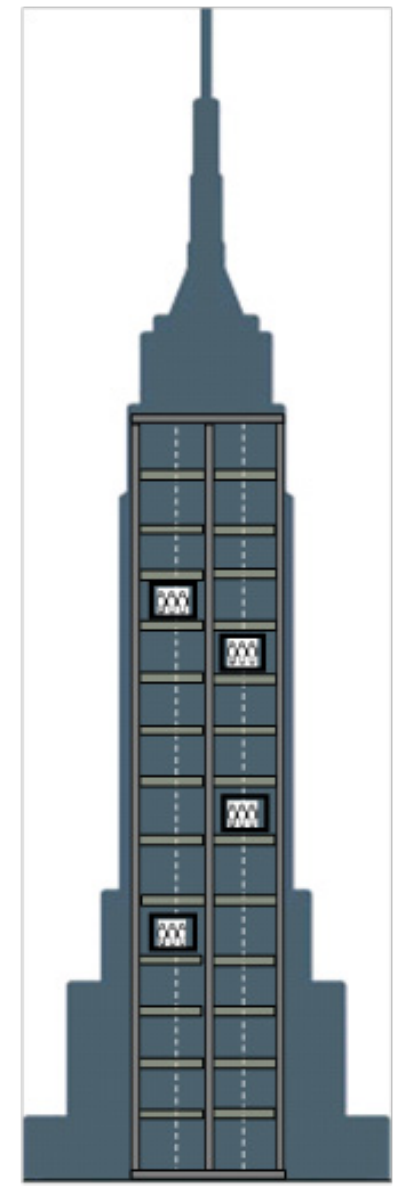


La compañía Thyssen Krupp patentó el primer ascensor twin [105] y viene apostando firmemente por este tipo de configuraciones que permitiría reducir significativamente los tiempos de espera de los pasajeros. Adicionalmente, permitiría conseguir un mejor equilibrio en los consumos de energía del sistema gracias a un mejor balance entre cabinas y contrapesos en el conjunto del sistema, todo ello utilizando un menor volumen de construcción y de espacio. La compañía instala estos ascensores con un sistema de control de preselección de destinos, donde el pasajero establece su viaje previamente a entrar en la cabina desde el rellano en el acceso [106].

\section{Clasificación y análisis de la litera- tura}

Las tablas 1, 2 y 3 recogen diversas clasificaciones de la literatura analizada y que se ha discutido en este artículo de revisión del estado del arte. Así, la Tabla 1 agrupa las referencias analizadas en función de las tareas acometidas en el sistema de transporte vertical, de acuerdo con la estructuración que se definía en las Figuras 4 y 5 . Por otra parte, las Tablas 2 y 3 se centran en el problema de despacho de ascensores. Así. la Tabla 2 muestra la clasificación de acuerdo con los criterios de optimización implementados y, por su parte, la Tabla 3 estudia y agrupa las referencias según el enfoque metodológico implementado para la resolución del citado problema de asignación de cabina a llamada de planta. Por último, la Figura 13 permite apreciar la intensidad en el empleo de los enfoques metodológicos según el criterio empleado para el proceso de asignación de cabina a llamada de plantas. La Figura 13 muestra cómo el principal criterio seguido ha sido el del tiempo medio de espera (AWT), empleándose para su resolución enfoques de lógica difusa y algoritmos genéticos, si bien, la utilización del consumo de energía como criterio viene ganando un interés significativo en los últimos tiempos.
Tabla 1 Clasificación de las referencias de acuerdo a acometidas en el sistema de transporte vertical

\begin{tabular}{|c|c|c|c|c|c|c|c|}
\hline \multirow{2}{*}{ REFERENCIAS } & \multicolumn{7}{|c|}{ TAREAS DE ACUERDO CON LOS SUBSISTEMAS DEL EGCS } \\
\hline & $\begin{array}{l}\text { Captu- } \\
\text { ra de } \\
\text { Datos }\end{array}$ & $\begin{array}{c}\text { Detección } \\
\text { Patrón de } \\
\text { Tráfico }\end{array}$ & $\begin{array}{c}\text { Despacho } \\
\text { de ascen- } \\
\text { sores }\end{array}$ & $\begin{array}{l}\text { Calibración } \\
\text { Parámetros }\end{array}$ & $\begin{array}{c}\text { Sector- } \\
\text { ización } \\
\text { Dinámica }\end{array}$ & $\begin{array}{c}\text { Sistemas } \\
\text { Dou- } \\
\text { ble-Deck }\end{array}$ & $\begin{array}{l}\text { Ascensores } \\
\text { Twin }\end{array}$ \\
\hline [19] [24] & $\sqrt{ }$ & & & & & & \\
\hline [25] & $\sqrt{ }$ & $\sqrt{ }$ & & & & & \\
\hline [33] [34] [61] & & $\sqrt{ }$ & $\sqrt{ }$ & & & & \\
\hline [12] [34] [108] & & $\sqrt{ }$ & & & & & \\
\hline $\begin{array}{c}\text { [36] [37] [38] } \\
{[39]}\end{array}$ & & & $\sqrt{ }$ & $\sqrt{ }$ & & & \\
\hline [35] & & & & $\sqrt{ }$ & & & \\
\hline $\begin{array}{c}{[11][15][40]} \\
{[41][42][47]} \\
{[48][49][50]} \\
{[59][60][64]} \\
{[65][67][68]} \\
{[69][70][73]} \\
{[75][76][79]} \\
{[80][81][82]} \\
{[83]}\end{array}$ & & & v & & & & \\
\hline $\begin{array}{c}{[87][88][89]} \\
\text { [90] [91] } \\
\end{array}$ & & & & & $\sqrt{ }$ & & \\
\hline $\begin{array}{c}{[92][93][94]} \\
{[98][102][103]}\end{array}$ & & & & & & V & \\
\hline
\end{tabular}


Pablo Cortés et al. / Dirección y Organización 65 (2018) 36-59

Tabla 3 Clasificación de las referencias de acuerdo al enfoque metodológico para la resolución del problema de despacho de ascensores

\begin{tabular}{|c|c|c|c|c|c|c|c|c|c|c|}
\hline \multirow{2}{*}{$\begin{array}{l}\text { REFEREN- } \\
\text { CIAS }\end{array}$} & \multicolumn{10}{|c|}{ METODOLOGÍA } \\
\hline & Memoria & $\begin{array}{l}\text { Lógica } \\
\text { Difusa }\end{array}$ & $\begin{array}{c}\text { Redes } \\
\text { Neuronales } \\
\text { (NN) }\end{array}$ & $\begin{array}{c}\text { Alg. } \\
\text { Genéticos } \\
\text { (GA) }\end{array}$ & $\begin{array}{l}\text { Otros Alg. } \\
\text { Bio-Inspirados } \\
\text { (ACO, PSO, } \\
\text { VS, Sistemas } \\
\text { Inmunes) }\end{array}$ & $\begin{array}{l}\text { Búsqueda } \\
\text { Tabú (TS) }\end{array}$ & $\begin{array}{l}\text { Sist. Mul- } \\
\text { ti-agente }\end{array}$ & $\begin{array}{c}\text { Árboles } \\
\text { Búsqueda }\end{array}$ & Redes Petri & $\begin{array}{l}\text { Program. } \\
\text { Dinámica }\end{array}$ \\
\hline [25] & $\sqrt{ }$ & & & & & & & & & \\
\hline [61] & $\sqrt{ }$ & & $\sqrt{ }$ & & & & & & & \\
\hline $\begin{array}{c}{[15][32]} \\
{[33][34]} \\
{[36][38]} \\
{[47][48]} \\
{[49][50]} \\
{[109]}\end{array}$ & & $\sqrt{ }$ & & & & & & & & \\
\hline $\begin{array}{c}{[37][41]} \\
{[42][67]} \\
{[68][69]} \\
{[70][73]} \\
{[102]}\end{array}$ & & & & $\sqrt{ }$ & & & & & & \\
\hline [39] & & $\sqrt{ }$ & $\sqrt{ }$ & & $\sqrt{ }$ & & & & & \\
\hline [64] [65] & & $\sqrt{ }$ & $\sqrt{ }$ & & & & & & & \\
\hline [81] & & & & $\sqrt{ }$ & & $\sqrt{ }$ & & & & \\
\hline [82] [83] & & & & & & & $\sqrt{ }$ & & & \\
\hline [11] & & & & & & & & $\sqrt{ }$ & & \\
\hline $\begin{array}{c}\text { [59] [60] } \\
{[94]}\end{array}$ & & & $\sqrt{ }$ & & & & & & & \\
\hline [76] & & & & & & & & & $\sqrt{ }$ & \\
\hline [75] & & & & & & & & & & $\sqrt{ }$ \\
\hline [103] & & & & $\sqrt{ }$ & $\sqrt{ }$ & & & & & \\
\hline $\begin{array}{c}{[78][79]} \\
{[80][88]} \\
{[89][90]} \\
{[91]}\end{array}$ & & & & & $\sqrt{ }$ & & & & & \\
\hline
\end{tabular}


Figura 13 Intensidad en el empleo de los distintos enfoques metodológicos según el criterio empleado para el despacho de ascensores

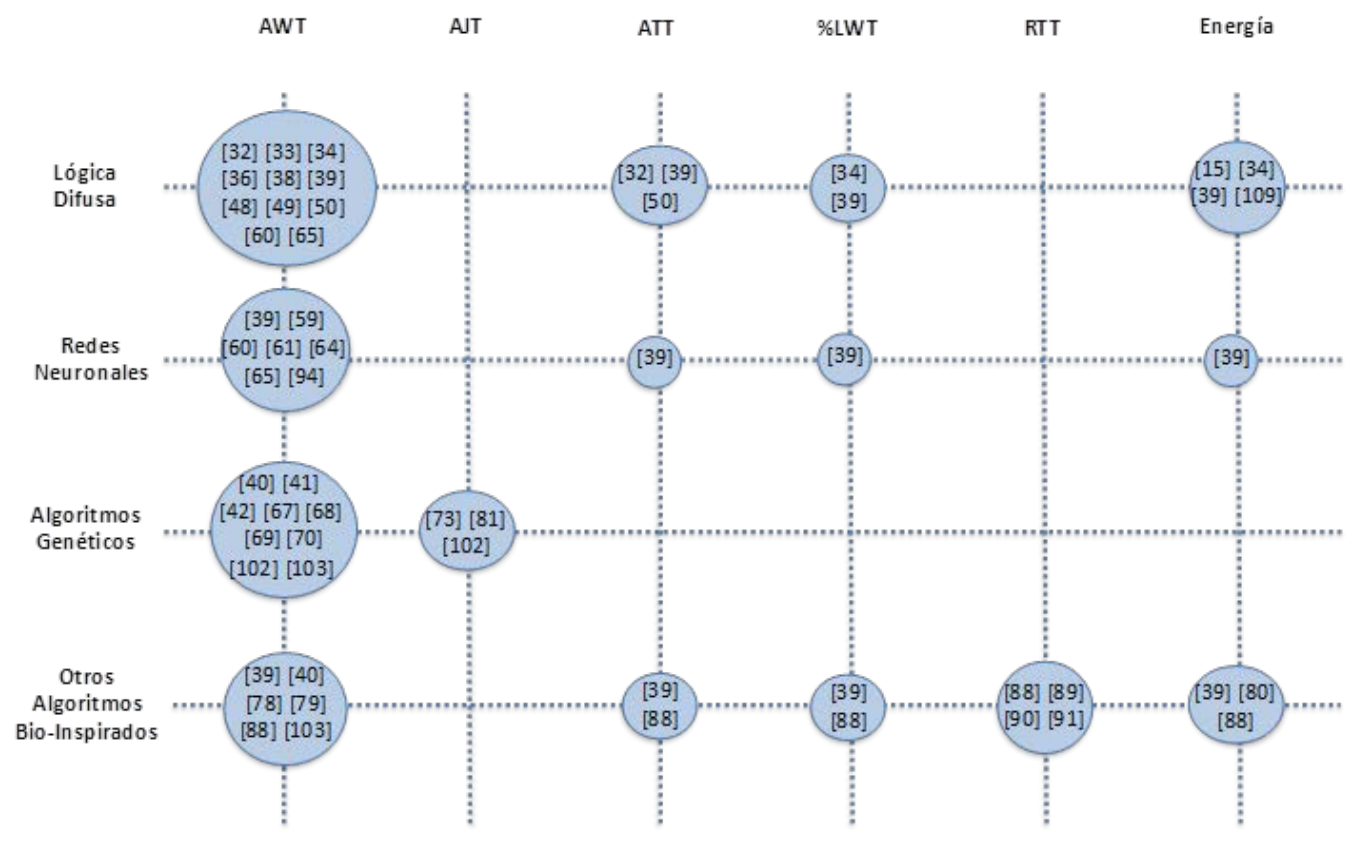

\section{Conclusión y líneas futuras de inves- tigación}

Esta revisión del estado del arte asociado a los problemas de transporte vertical constituye, probablemente, la primera que se realiza en español, a la vez que es también una de las primeras desarrolladas en la literatura científica, en general. Así, la Tabla 1 supone una clasificación interesante de las contribuciones más significativas asociadas a los principales problemas de transporte vertical.

El objetivo de este estudio es que pueda servir como punto de partida para el inicio de una investigación en materia de transporte vertical. Actualmente, la mayoría de las investigaciones ha tenido como objetivo principal la optimización del problema de asignación de cabinas a llamadas de planta, lo que proporciona una mejor calidad de servicio (las Tablas 2 y 3 muestran un resumen de las numerosas contribuciones en este ámbito). El enfoque para proceder a tal asignación puede establecerse desde diferentes criterios de decisión (Tabla 2) y enfocarse bajo numerosas metodologías que son familiares para los investigadores de la disciplina de la Ingeniería de Organización (Tabla 3). No obstante, los EGCS pueden realizar otras tareas secundarias para mejorar el despacho de ascensores que no deben desatenderse, como son reducir la incertidumbre mediante una captura adecuada de datos o mediante la detección de patrones de tráfico, así como la calibración de los parámetros de los diferentes subsistemas del EGCS con objeto de mejorar rendimiento del sistema.

En el ámbito del despacho de ascensores, se aprecia cómo han venido surgiendo nuevos criterios, en ocasiones introducidos por los propios gestores de los edificios como el caso del ahorro energético. Así, estos criterios, ya se consideran habitualmente junto a los parámetros clásicos de desempeño basados en tiempos de espera o vueltas dadas al edificio para las fases de uppeak.

En el plano de las metodologías para la resolución de problemas, la lógica difusa, dada la imperfección de los datos, se muestra como una herramienta adecuada. En este sentido, las redes neuronales deberían también recuperar un espacio perdido en los últimos años. Los algoritmos basados en inteligencia artificial también pueden aportar procesos eficientes de búsqueda de soluciones en el espacio de soluciones y han sido ensayados con entusiasmo. Cabe señalar, no obstante, que la mayoría de estos enfoques son iterativos y, por lo tanto, pueden tomar mucho tiempo de cálculo. La calibración y elección de los parámetros se convierte en un problema crítico además en estos casos. Estos parámetros deben seleccionarse atendiendo, no tanto a la precisión del algoritmo sino al tiempo disponible entre diferentes eventos. Es decir, el tiempo necesario para proceder a asignar una llamada de planta entre distintos eventos producidos en el sistema de transporte vertical. Así, para casos reales, una alternativa puede ser detener el algoritmo, mediante una consigna de control, previamente al instante en que sea necesario dar respuesta al siguiente evento.

Sin embargo, el desafío más relevante en este campo parece relacionarse con el vínculo entre el Internet de las cosas y los EGCS. Las posibilidades de computación ubicua en la cabina, junto con la capacidad de integrar la inteligencia ambiental en el sistema de transporte vertical (mediante el despliegue de sensores y electrónica de hueco) se han convertido ya en un tema abierto y desafiante. Esta inteligencia ambiental conducirá a un entorno más amigable con el usuario; un apoyo a los servicios de operación del sistema más eficientes y una mejora de las interacciones hu- 
mano-máquina que conduzcan a un mayor empoderamiento del pasajero. La construcción de un verdadero entorno de inteligencia ambiental necesitará de técnicas avanzadas de computación y comunicación ubicua, interfaces de usuario inteligentes, la disposición de sistemas empotrados para el reconocimiento de patrones de tráfico o cámaras y dispositivos para el reconocimiento patrones mediante visión, entre otros. Siguiendo esta línea, los temas relevantes que se incorporarán en un futuro próximo como la inteligencia artificial distribuida, la visión o el reconocimiento de patrones para el control y monitorización de grupos de ascensores, las interfaces hombre-máquina, la computación y la comunicación ubicua, y los sistemas embebidos deberían atraer la atención de la investigación a llevar a cabo en el campo del transporte vertical.

\section{Agradecimientos}

Son numerosos los agradecimientos a señalar que han permitido la realización de este trabajo. En primer lugar, hay que destacar la colaboración de la empresa MP Ascensores que contribuyó a la creación de esta línea de trabajo en su origen, por medio de una solicitud de transferencia de tecnología en el año 2001. Desde aquel momento se estabilizó una línea de investigación que dio lugar a siete contratos de I+D concatenados y continuados de investigación aplicada e industrial, de la que se distingue el registro y licencia de tres patentes industriales [107-109]. En segundo lugar, en esta línea de investigación destacan los dos proyectos financiados por el Plan Nacional de I+D (Ref.: TI-331/2002 y DPI201015352) y el proyecto de Excelencia financiado por la Consejería de Innovación, Ciencia y Empresa de la Junta de Andalucía (Ref.: P07-TEP-02832).

Por último, corresponde señalar que las figuras 1, 2, 10 y 11 se han reproducido bajo la licencia Creative Commons License Wikimedia y que las imágenes de botoneras mostradas en la figura 4 son cortesía de la empresa MP Ascensores.

\section{Referencias}

1. E Graves Otis 1861 "Improvement in hoisting apparatus"; US 31128 A.

2. Landmark Elevator Inc. (2016). History of Elevator Technology. https://landmarkelevator.com/history-of-elevator-technology/

3. M Thornton (2005). "Skyscrapers and business cycles". The Quarterly Journal of Austrian Economics. Vol. 8 no. 1. pp. 51-74.

4. P Cortés, J Guadix, J Muñuzuri. "A State of the Art on the most relevant patents in vertical transportation in buildings”. Recent Patents on Computer Science, vol. 2 no. 2, pp. 96-110. 2009
5. JR Fernández, P Cortés (2015). A Survey of Elevator Group Control Systems for Vertical Transportation: A Look at Recent Literature. IEEE Control Systems Magazine, Vol. 35, No. 4, pp. 38-55

6. P Cortés, J Muñuzuri, L Onieva. "Design and analysis of a tool for planning and simulating dynamic vertical transport”. Simulation: Transactions of the Society for Modelling and Simulation International Vol. 82, no. 4, pp. 255-274, 2006.

7. G Barney. Elevator handbook Theory and Practice, Spon Press, London 2003.

8. T Susi, J Sorsa, ML Siikonen (2005), "Passenger Behaviour in Elevator Simulation," Elevatori, Vol. 34, pp. 28-37.

9. MAT Valk, DJ Sirag. "Elevator dispatching with balanced passenger perception of waiting" U.S. Patent No. 7,475,757. 13 Jan. 2009.

10. J Sorsa (2017). Optimization Models and Numerical Algorithms for an Elevator Group Control System. Ph.D Dissertation Aalto University.

11. M Hamdi, D Mulvaney. "Prioritised A* search in real-time elevator dispatching”. Control Engineering Practice 2007 vol. 15 no. 2 pp. 219-230.

12. P Cortés, J R Fernández, J Guadix, J Muñuzuri. "Fuzzy logic based controller for peak traffic detection in Elevator systems”. Journal of Computational and Theoretical Nanoscience no. 9 pp. 310-318.2012

13. Z Hu, Y Liu, Q Su, J Huo. "A multi-objective genetic algorithm designed for energy saving of the elevator system with complete information”. IEEE International Energy Conference and Exhibition, EnergyCon2010. art. no. 5771661 pp. 126-130.

14. T Tyni, J Ylinen, M Matela, T Rintala. 2006; US20067140472.

15. JR Fernández, P Cortés, J Guadix, J Muñuzuri. “Dynamic fuzzy logic elevator group control system for energy optimization”. International Journal of Information Technology \& Decision Making vol. 12, no. 3, pp. 591617, 2013.

16. MZ Hasan, R Fink, MR Suyambu, MK Baskaran. "Assessment and improvement of intelligent controllers for elevator energy efficiency". IEEE International Conference on Electro Information Technology 2012; art. no. 6220727 . 
17. CIBSE Guide D: Transportation systems in buildings, Chartered Institution of Building Services Engineers. London 2010.

18. K Thangavelu. 1991 "Artificial intelligence based crowd sensing system for elevator car assignment"; US5022497.

19H Kulju, A Vurri. "Procedure and means for counting objects within a given area”. Kone Elevator December 1987; GB Patent 2191358.

20J Kim, B Moon. “Adaptive elevator group control with cameras". IEEE Transactions on Industrial Electronics vol. 48 no. 2 pp. $377-382.2001$

21L Lin, Z Xiong, M A Finn, P Y Peng, M Atalla, P Kang, M Misra, C M Netter. 2008: GB2447829(A).

22N Yamaguchi, T Suzuki. 2008 "Functional variable remote monitoring system and remote monitoring method for elevator"; JP2008081299(A).

23R Deplazes, E Cortona. 2006 “Three-dimensional monitoring in the area of an elevator by means of a three-dimensional sensor"; US20060037818.

24J Sorsa, H Ehtamo, JM Kuusinen, M Ruokokoski, ML Siikonen (2017). Modeling uncertain passenger arrivals in the elevator dispatching problem with destination control. Optimization Letters, 1-15.

25K Thangavelu, "Artificial intelligence based learning system predicting peak-period times for elevator dispatching”. Otis Elevator Company 1993; US5241142.

26N Kameli 1993 "Elevator traffic predictions using historical data checked for certainty"; US5272288.

27N Kameli 1994 "Predictor elevator for traffic during peak conditions"; US5276295.

28K Thangavelu 1991 “Artificial Intelligence based learning system predicting "Peak-Period" times for elevator dispatching"; US5035302.

29Y Xu, F Luo. "Pattern recognition of traffic flows in elevator group control systems based on SVM". Journal of South China University of Technology2005.

30F Luo, YG Xu, J Z Cao. "Elevator traffic flow prediction with least squares supports vector machines". Fourth Int Conf on Machine Learning and Cybernetics 2005 vol. 18-21 pp. 4266-4270.
31. 程建，林超，苏靖峰，刘罕. 2008 “Method and apparatus for controlling elevator button”; CN101456501B.

32. ML Siikonen, J Leppala. "Elevator traffic pattern recognition” Proceedings of the 4th IFSA Congress1991 pp.195-198.

33. S Hikita, K Komaya. "A new elevator group-supervisory control system using fuzzy rule-base”. J. Soc. Instrum Control Eng 1989 vol. 25 pp. 99-104.

34. C Kim, O Jeong. “Group management control method for elevator system employing traffic flow estimation by fuzzy logic using variable value preferences and decisional priorities". LG Industrial Systems Co1997: US5679932.

35. T Tobita, A Fujino, K Segawa, K Yoneda, Y Ichikawa. "A parameter tuning method for an elevator group control system using a genetic algorithm”. Proceedings of the IEEE 22nd International Conference on Industrial Electronics, Control and Instrumentation 1996 vol. 2 pp. 823-828.

36. S Oh, D Park. "Self-tuning fuzzy controller with variable universe of discourse". Proceedings of the IEEE International Conference on Systems Man and Cybernetics 1995 pp. 2628-2632.

37. T Tyni, J Ylinen. "Evolutionary bi-objective optimisation in the elevator car routing problem”. European Journal of Operational Research 2004 vol. 169 pp. 960977.

38. J Jamaludin, N Rahim, W Hew. "An Elevator Group Control System with a Self-Tuning Fuzzy Logic Group Controller". IEEE Transactions on Industrial Electronics 2010 vol. 57 no. 12 pp. 4188-4198.

39. J Liu, Y Liu. "Ant Colony Algorithm and Fuzzy Neural Network based Intelligent Dispatching Algorithm of An Elevator Group Control System”. IEEE International Conference on Control and Automation2007 pp. 23062310 .

40. B Bolat, O Altun, P Cortés, YE Yildiz, AO Topal (2017). A Comparison of Metaheuristics for the Allocation of Elevators to Calls in Buildings, pages: 519-529. Politeknik Dergisi, 20(3).

41. P Cortes, J Larrañeta, L Onieva (2003) A Genetic Algorithm for Controlling Elevator Group Systems. In: Mira J., Álvarez J.R. (eds) Artificial Neural Nets Problem Solving Methods. IWANN 2003. Lecture Notes in Computer Science, vol 2687. Springer, Berlin, Heidelberg. 
42. P Cortés, J Larrañeta, L Onieva (2004). “Genetic algorithm for controllers in elevator groups: analysis and simulation during lunchpeak traffic". Applied Soft Computing vol.4 pp. 159-174.

43. S Kuzunuki, Y Morita, K Yoneda, T Ueshima, T Tobita. 1990; US4947965.

44. T Tyni, J Ylinen. 1999 "Method and apparatus for allocating landing calls in an elevator group"; US5932852.

45. D Nikovski, M Brand. 2004 "Method for scheduling elevator cars using pairwise delay minimization”; WO04113216.

46. M J Atalla, AC Hsu, PB Luh, GG Luther, B Xiong 2006 "Group elevator scheduling with advanced traffic information”; WO/2006/113598 (A2).

47. J Fernández, P Cortés, J Muñuzuri, J Guadix. "Dynamic Fuzzy Logic Elevator Group Control System with Relative Waiting Time Consideration”. IEEE Transactions on Industrial Electronics 2014 vol. 61 no. 9 pp. 49124919.

48. MD Sale, VC Prakash, V. C. (2017). Dynamic Scheduling of Elevators with Reduced Waiting Time of Passengers in Elevator Group Control System: Fuzzy System Approach. In Innovations in Computer Science and Engineering (pp. 339-345). Springer, Singapore.

49. C Kim, K Seong, H Lee-kwang. "Design and implementation of a fuzzy elevator group control system”. Proceedings of the IEEE Transactions on systems man and Cybernetics 1998 vol. 28 no. 3 pp. 277-287.

50. T Ishikawa, A Miyauchi, M Kaneko. "Supervisory control for elevator group by using fuzzy expert system which also addresses traveling time". Proceedings of the IEEE International Conference on Industrial Technology 2000 vol. 2 no. 1 pp. 87-94.

51. N Imasaki, S Kubo, S Nakai, T Yoshitsugu, K Jun-Ichi, $\mathrm{T}$ Endo. "Elevator group control system tuned by a fuzzy neural network applied method". Proceedings of IEEE International Conference on Fuzzy Systems 1995. International Joint Conference of the Fourth IEEE International Conference on Fuzzy Systems and The Second International Fuzzy Engineering Symposium 1995 vol. 4 pp. $1735-1740$.

52. M Amano 1993 "Elevator control apparatus using evaluation factors and fuzzy logic"; US5233138.
53. J O Kim 1997 “Group management control method for elevator system employing traffic flow estimation by fuzzy logic using variable value preferences and decisional priorities"; US5679932.

54. DJ Jr Sirag 1993 "Elevator car assignment conditioned on minimum criteria”; US5260526.

55. DJ Jr Sirag 1993 "Using fuzzy logic to determine elevator car assignment utility”; US5248860.

56. K Sasaki, K Yokota, H Hattori, N Sata 1991 "Method and apparatus for controlling a group of elevators using fuzzy rules”; US5022498.

57. JA Stanley, H Honma, D S Williams, T Mori, P Simcik 2007 "Elevator car dispatching including passenger destination information and a fuzzy logic algorithm"; US20070045052(A1).

58. MM Rashid, B Kasemi, A Faruq, AZ Alam. "Design of fuzzy based controller for modern elevator group with floor priority constraints". 4th International Conference on Mechatronics: Integrated Engineering for Industrial and Societal Development, ICOM'11 2011. Art. no. 5937196.

59. T Shintaro. "Elevator control apparatus using neural net” 1991; UKGB245 997A.

60. S Kenji, M Sandor, N Masami. "Elevator group supervisory control system using neural network". Elevator World 1996 vol. 44 pp. 81-86.

61. C Imrak, G Barney. “Application of neural networks on traffic control". Proceedings of ELEVCON 1998 pp. 140-148.

62. BL Whitehall, Jr DJ Sirag,B A Powell 1997 "Elevator control neural network"; US5672853.

63. BL Whitehall, TM Christy, B A Powell 1999 "Method for continuous learning by a neural network used in an elevator dispatching system"; US5923004.

64. I Naoki, K Susumu, N Shoji. "Elevator group control system tuned by a fuzzy neural network applied method”. IEEE International Conference on Fuzzy Systems 1995 pp. $1735-1740$.

65. C Gu, G Zhao, Z Liu. "Adaptive fuzzy logical elevator group control system based on artificial neural net". Computer Measurement and Control vol.11 pp.947-949. 2003. 
66. M Nakagawa, K Sasaki, S Markon, I Nagashima, T Midoritani, T Tanabe, N Ohta 1998 "Elevator group supervisory control system”; US5767461.

67. S Mabu, L Yu, J Zhou, S Eto, K Hirasawa. "A double-deck elevator systems controller with idle cage assignment algorithm using genetic network programming”. Journal of Advanced Computational Intelligence and Intelligent Informatics 2010 vol. 114 no. 5 pp. 487 496.

68. J Alander, T Tyni, J Ylinen. "Elevator group control using distributed genetic algorithm”. Proceedings of the international conference on artificial neural nets and genetic algorithms (ICANNGA95) 1995 pp. 400-403.

69. A Miravete. "Genetics and intense vertical traffic", Elevator World 1999 vol. 47 no 7 pp. 118-120.

70. A Fujino, T Tobita, K Segawa, K Yoneda, A Togawa. "An elevator group control system with floor-attribute control method and system optimization using genetic algorithms”. IEEE Trans Industrial Elect vol. 44 no.4 pp. 546-552. 1997

71. S Tsuji 1998 "Group managing system for elevator cars"; US5780789.

72. EL Koh, JO Kim, PH Hahn 1999 “Group management control method for elevator”; US6000504.

73. B Bolat, P Cortés, E Yalcin, M Alisverisci. “Optimal car dispatching for elevator groups using genetic algorithms”. Intelligent Automation and Soft Computing vol. 16 no. 1 pp. 89-99. 2010

74. ML Siikonen, J Ylinen 2005 "Method for controlling an elevator system"; WO2005100223.

75. D Pepyne, C Cassandras. "Optimal dispatching control for elevator systems during uppeak traffic". IEEE Transactions on Control System Technology 1997 vol. 5 no.6 pp. 629-643.

76. Z Qun, S Ding, C Yu, L Xiaofeng, "Elevator group control system modeling based on object oriented petri net”. Elevator World vol. 49 no. 8 pp. 99-105.2001.

77. K Verma, J Goubault-Larrecq. "Karp-Miller trees for a branching extension of VASS”. Discrete Mathematics and Theoretical Computer Science vol. 7 pp. 217230.2005 .

78. B Bolat, O Altun, P Cortés 2012. “A particle swarm optimization algorithm for optimal car-call allocation in elevator group control systems”. Applied Soft Computing Journal vol. 13 no. 5 pp. 2633-2642.
79. P Cortés, L Onieva, J Muñuzuri, J Guadix 2013 “A viral system algorithm to optimize the car dispatching in elevator group control systems of tall buildings”. Computers and Industrial Engineering vol. 64 no. 1 pp. 403-411.

80. Zhang, J. L., Tang, J., Zong, Q., \& Li, J. F. (2010, November). Energy-saving scheduling strategy for elevator group control system based on ant colony optimization. In Information Computing and Telecommunications (YC-ICT), 2010 IEEE Youth Conference on (pp. 37-40). IEEE.

81. B Bolat, P Cortés. "Genetic and tabu search approaches for optimizing the hall call Car allocation problem in elevator group systems”. Applied Soft Computing vol.11 no. 2 pp. $1792-1800.2011$.

82. Y Ogoshi, H Kimura, S Hirose, N Osato. "Elevator group control system using multiagent system”, Systems and Computers in Japan. vol. 34 pp. 45-58.2003.

83. D Muñoz, C Llanos, M Ayala-Rincón, R Van Els, R Almeida. "Implementation of dispatching algorithms for elevator systems using reconfigurable architectures". Engineering Applications of Artificial Intelligence vol. 21 no. 8 pp. $1309-1320.2008$.

84. ZT Wang, DF Ji, F Qiao, QD Wu. "Modeling and scheduling elevator group control system based on MAS and negotiation mechanism of agents”. Control and Decisionvol.22 no. 10 pp. 1184-1188. 2007.

85. Al-Sharif, L., Yang, Z. S., Hakam, A., \& Abd Al-Raheem, A. (2018). Comprehensive analysis of elevator static sectoring control systems using Monte Carlo simulation. Building Services Engineering Research and Technology, 0143624417752644.

86. W Chan, A So, K Lam. "Dynamic zoning in elevator traffic control". Proceedings of International Conference on Elevator Technologies, Hong Kong, China 1995 pp. 132-140.

87. A So, W Chan. "Comprehensive dynamic zoning algorithms”. Elevator World 1997 vol. 45 no. 8 pp. 99-109.

88. Z Li. "Traffic based on artificial immune algorithm research on dynamic zoning of elevator traffic based on artificial immune algorithm". Proceedings of Control Automation Robotics and Vision Conference, Guangzhou, China 2004 vol. 3 pp. 2170-2171.

89. Y Xu, F Luo, X Lin. "Hybrid destination registration elevator group control system with artificial immune optimization algorithm”. Proceedings of the 8th World Congress on Intelligent Control and Automation, WCICA 2010 pp. 5067-5071. 
90. Z Li, HZ Tan, Y Zhang. "Particle swarm optimization applied to vertical traffic scheduling in buildings". Lecture Notes in Comp Sci pp. 831-838. 2007.

91. L Fei, Z Xiaocui, X Yuge. “A new hybrid elevator group control system scheduling strategy based on particle swarm simulated annealing optimization algorithm”. Proceedings of the 8th World Congress on Intelligent Control and Automation, WCICA 2010 pp. 5121-5124.

92. Janne Sorsa, Mirko Ruokokoski Multiple objectives and system constraints in double-deck elevator dispatching. Proceedings of FORS40 - 2013 Lappeenranta, Finland ISBN 978-952-265-436-6, pp. 48-51

93. F Nowak, J Luce 1986 "Multicompartment elevator call assigning"; US4632224.

94. K Sasaki, S Markon, M Makagawa. "Elevator group supervisory control system using neural networks". Elevator World 1996.

95. T Mori, Z Bahjat, J Stanley, M Ross, M Sahara, H Honma. 2001 "Control system and control method for double-deck elevator”; US20010032756.

96. M Kostka, K Steinmann 2003 "Method of controlling elevator installation with multiple cars"; US6508333.

97. ML Siikonen 2001 "Procedure for control of an elevator group consisting of double-deck elevators which optimizes passenger journey time"; US6237721.

98. ML Siikonen 2002 "Double-deck elevator group controller for call allocation based on monitored passenger flow and elevator status"; US6401874.

99. ML Siikonen, J Sorsa. "Optimal control of double deck elevator group using genetic algorithm”, KONE Corporation 2002.

100 J Ylinen, TTyni 2001 "Genetic procedure for multi-deck elevator call allocation": US6293368.
101 M Ruokokoski, J Sorsa, ML Siikonen, H Ehtamo (2016). Assignment formulation for the Elevator Dispatching Problem with destination control and its performance analysis. European Journal of Operational Research, 252(2), 397-406.

102 J Sorsa, M Siikonen, H Ehtamo. "Optimal control of double deck elevator group using genetic algorithm". International transactions in operational research vol. 10 pp. 103-114.2003.

103 L Yu, J Zhou, S Mabu, K Hirasawa, J Hu, S Markon. "Elevator group control system using genetic network programming with ACO considering transitions”. SICE Annual Conference, Kagawa University, Japan 2007 pp. 1330-1336.

104K Hirasawa, T Eguchi, J Zhou, L Yu, J Hu, S Markon. "A double-deck elevator group supervisory control system using genetic network programming". IEEE Trans Syst Man Cybernet Part C: Applications and Reviews vol. 38 no. 4 pp. 535-550. 2008.

105RS Smith, RW Huff, RD Peters, B Powell, G Thumm 2005 "Twin elevator systems"; US 7841450 B2

106 Thyssenkrupp TWIN_http://elevation.wikia.com/wiki/ Thyssenkrupp_TWIN.

107 P Cortés, JR Fernández, L Onieva, J Muñuzuri, J Guadix, P Fernández-Montero de Espinosa 2007 "System and method for selecting a system of elevators"; WO2007ES00045 20070129 PCT/2007/000045

108P Cortés, JR Fernández, L Onieva, J Muñuzuri, J Guadix, P Fernández-Montero de Espinosa 2010 “Dynamic controller for detecting traffic patterns by means of fuzzy logic"; WO/2010/109035 PCT/ES2010/000122

109P Cortés, JR Fernández, L Onieva, J Muñuzuri, J Guadix, P Fernández-Montero de Espinosa 2010 "Controlador difuso de grupo de ascensores para la optimización del consumo energético"; ES201000497 